\title{
On a class of left metacyclic codes
}

\author{
Yonglin Cao, Yuan Cao, Fang-Wei Fu and Jian Gao
}

\begin{abstract}
Let $G_{(m, 3, r)}=\left\langle x, y \mid x^{m}=1, y^{3}=1, y x=x^{r} y\right\rangle$ be a metacyclic group of order $3 m$, where $\operatorname{gcd}(m, r)=1$, $1<r<m$ and $r^{3} \equiv 1(\bmod m)$. Then left ideals of the group algebra $\mathbb{F}_{q}\left[G_{(m, 3, r)}\right]$ are called left metacyclic codes over $\mathbb{F}_{q}$ of length $3 m$, and abbreviated as left $G_{(m, 3, r)}$-codes. A system theory for left $G_{(m, 3, r)}$-codes is developed for the case of $\operatorname{gcd}(m, q)=1$ and $r \equiv q^{\epsilon}$ for some positive integer $\epsilon$, only using finite field theory and basic theory of cyclic codes and skew cyclic codes. The fact that any left $G_{(m, 3, r)}$-code is a direct sum of concatenated codes with inner codes $\mathcal{A}_{i}$ and outer codes $C_{i}$ is proved, where $\mathcal{A}_{i}$ is a minimal cyclic code over $\mathbb{F}_{q}$ of length $m$ and $C_{i}$ is a skew cyclic code of length 3 over an extension field of $\mathbb{F}_{q}$. Then an explicit expression for each outer code in any concatenated code is provided. Moreover, the dual code of each left $G_{(m, 3, r)}$-code is given and self-orthogonal left $G_{(m, 3, r)}$-codes are determined.
\end{abstract}

\section{Index Terms}

Left metacyclic code, Cyclic code, Skew cyclic code, Dual code, Self-orthogonal code.

\section{INTRODUCTION}

$\mathbf{L}$ ET $\mathbb{F}_{q}$ be a finite field of cardinality $q$ and $G$ a group of order $n$. The group algebra $\mathbb{F}_{q}[G]$ is a vector space over $\mathbb{F}_{q}$ with basis $G$. Addition, multiplication with scalars $c \in \mathbb{F}_{q}$ and multiplication are defined by: for any $a_{g}, b_{g} \in \mathbb{F}_{q}$,

$$
\begin{gathered}
\sum_{g \in G} a_{g} g+\sum_{g \in G} b_{g} g=\sum_{g \in G}\left(a_{g}+b_{g}\right) g, \\
c\left(\sum_{g \in G} a_{g} g\right)=\sum_{g \in G} c a_{g} g, \\
\left(\sum_{g \in G} a_{g} g\right)\left(\sum_{g \in G} b_{g} g\right)=\sum_{g \in G}\left(\sum_{u v=g} a_{u} b_{v}\right) g .
\end{gathered}
$$

Then $\mathbb{F}_{q}[G]$ is an associative $\mathbb{F}_{q}$-algebra with identity $1=1_{\mathbb{F}_{q}} 1_{G}$ where $1_{\mathbb{F}_{q}}$ and $1_{G}$ are the identity elements of $\mathbb{F}_{q}$ and $G$ respectively. Readers are referred to [?] for more details on group algebra.

Let $G=G_{(m, s, r)}=\left\langle x, y \mid x^{m}=1, y^{s}=1, y x=x^{r} y\right\rangle$ where $\operatorname{gcd}(m, r)=1$ and $r^{s} \equiv 1(\bmod m)$. Then $G$ is called a metacyclic group of order sm. Sabin and Lomonaco [?] provided a unique direct decomposition of the $\mathbb{F}_{2}$-algebra $\mathbb{F}_{2}\left[G_{(m, n, r)}\right]$ to minimal two-sided ideals (central codes) and described a technique to decompose minimal central codes to a direct sum of $n$ minimal left ideals (left codes) and gave an algorithm to determine these minima left codes. They discovered several good metacyclic codes and they expressed the hope that more "good" and perhaps even "best" codes may be discovered among the ideals of non-abelian group rings. Recently, Olteanu et al [?] provided algorithms to construct minimal left group codes and rediscovered some best codes. These are based on results describing a complete set of orthogonal primitive idempotents in each Wedderburn component of a semisimple finite group algebra $\mathbb{F}_{q}[G]$ for a large class of groups $G$. For example, by use of the computer algebra system GAP and the packages GUAVA and Wedderga some optimal codes and non-abelian group codes were obtained:

$\diamond$ A linear $[27,18,2]$-code constructed by a left ideal in $\mathbb{F}_{2}[G]$, where $G=\left\langle x, y \mid x^{9}=1, y^{3}=1, y x=x^{4} y\right\rangle($ [?, Example 1]).

$\diamond$ A best linear $[20,4,8]$-code constructed by a left ideal in $\mathbb{F}_{3}[G]$, where $G=\left\langle x, y \mid x^{5}=1, y^{4}=1, y x=x^{2} y\right\rangle$ ( [?, Example 3]).

Yonglin Cao is with School of Sciences, Shandong University of Technology, Zibo, Shandong 255091, China. e-mail: ylcao@sdut.edu.cn. Yuan Cao is with College of Mathematics and Econometrics, Hunan University, Changsha 410082, China. e-mail: yuan_cao@hnu.edu.cn. Fang-Wei Fu is with Chern Institute of Mathematics and LPMC, Nankai University, Tianjin 300071, China. e-mail: fwfu@nankai.edu.cn. Jian Gao is with School of Sciences, Shandong University of Technology, Zibo, Shandong 255091, China. e-mail: dezhougaojian@163.com. Manuscript received , 2016; revised , . 
$\diamond$ A non-abelian group code $[55,10,22]$-code constructed by a left ideal in $\mathbb{F}_{2}[G]$, where $G=\langle x, y| x^{11}=$ $\left.1, y^{5}=1, y x=x^{3} y\right\rangle([?$, Example 4$])$.

For any $\mathbf{a}=\left(a_{0,0}, a_{1,0}, \ldots, a_{m-1,0}, a_{0,1}, a_{1,1}, \ldots, a_{m-1,1}, a_{0, s-1}, a_{1, s-1}, \ldots, a_{m-1, s-1}\right) \in \mathbb{F}_{q}^{s m}$, define

$$
\Psi(\mathbf{a})=\left(1, x, \ldots, x^{m-1}\right) M_{\mathbf{a}}\left(\begin{array}{c}
1 \\
y \\
\cdots \\
y^{s-1}
\end{array}\right)
$$

where $M_{\mathbf{a}}=\left(\begin{array}{cccc}a_{0,0} & a_{0,1} & \ldots & a_{0, s-1} \\ a_{1,0} & a_{1,1} & \ldots & a_{1, s-1} \\ \ldots & \ldots & & \\ a_{m-1,0} & a_{m-1,1} & \ldots & a_{m-1, s-1}\end{array}\right)$. Then $\Psi$ is an $\mathbb{F}_{q}$-linear isomorphism from $\mathbb{F}_{q}^{\text {sm }}$ onto $\mathbb{F}_{q}\left[G_{(m, s, r)}\right]$. As in [?] and [?] a nonempty subset $C$ of $\mathbb{F}_{q}^{s m}$ is called a left metacyclic code (or left $G_{(m, s, r)}$-code for more precisely) over $\mathbb{F}_{q}$ if $\Psi(C)$ is a left ideal of the $\mathbb{F}_{q}$-algebra $\mathbb{F}_{q}\left[G_{(m, s, r)}\right]$. From now on, we will identify $C$ with $\Psi(C)$ for convenience.

In this paper, we focus our attention on the case of $s=3$ in the metacyclic group $G_{(m, s, r)}$ and $r=q^{\epsilon}$ for some positive integer $\epsilon$.

Compared with the known theory for cyclic codes over finite fields, literatures related with metacyclic codes were involved too much group algebra language and techniques. A system and elementary theory for left metacyclic codes over finite fields have not been developed fully to the best of our knowledge. In this paper, we try to achieve the following goals:

- Develop a system theory for left $G_{(m, 3, r)}$-codes using an elementary method. Specifically, only finite field theory and basic theory of cyclic codes and skew cyclic codes are involved, and it does not involve any group algebra language and technique.

- Provide a clear expression for each left $G_{(m, 3, r)}$-code over $\mathbb{F}_{q}$ and give a formula to count the number of all such codes.

- Give an explicit expression of the dual code for each left $G_{(m, 3, r)}$-code over $\mathbb{F}_{q}$ and determine its selforthogonality.

Using the expression provided, one can list all distinct left $G_{(m, 3, r)}$-codes for specific $m$ and $q$ (not too big) conveniently and easily, design left $G_{(m, 3, r)}$-codes for their requirements and encode the presented codes directly.

The present paper is organized as follows. In section 2, we prove that any left $G_{(m, 3, r)}$-code over $\mathbb{F}_{q}$ is a direct sum of concatenated codes with inner codes $\mathcal{A}_{i}$ and outer codes $C_{i}$ for the case of $\operatorname{gcd}(n, q)=1$ and $r \equiv q^{\epsilon}$ $(\bmod m)$ for some positive integer $\epsilon$, where $\mathcal{A}_{i}$ is a minimal cyclic code over $\mathbb{F}_{q}$ of length $m$ and $C_{i}$ is a skew $\theta_{i}$-cyclic code over $K_{i}$ of length 3 , i.e., left ideals of the ring $K_{i}\left[y ; \theta_{i}\right] /\left\langle y^{3}-1\right\rangle$, where $K_{i}$ is an extension field of $\mathbb{F}_{q}$ and $\theta_{i} \in \operatorname{Aut}_{\mathbb{F}_{q}}\left(K_{i}\right)$ satisfying $\theta_{i}^{3}=\mathrm{id}_{K_{i}}$. In Section 3, we give a precise description for skew $\theta_{i}$-cyclic codes over $K_{i}$ of length 3 . Hence all distinct left $G_{(m, 3, r)}$-codes over $\mathbb{F}_{q}$ can be determined by their concatenated structure. In Section 4, we give the dual code of each left $G_{(m, 3, r)}$-code over $\mathbb{F}_{q}$ precisely and determine all selforthogonal left- $G_{(m, 3, r)}$-codes. Finally, we list all distinct 541696 left $G_{(14,3,9)}$-codes and all 3364 self-orthogonal left $G_{(14,3,9)}$-codes over $\mathbb{F}_{3}$ in Section 5 .

\section{The CONCATENATED STRUCTURE OF LEFT $G_{(m, 3, r)}$-CODES OVER $\mathbb{F}_{q}$}

In this section, we overview properties for concatenated structure of linear codes and determine the concatenated structure of left $G_{(m, 3, r)}$-codes over $\mathbb{F}_{q}$.

Let $B$ be a linear $\left[n_{B}, k_{B}, d_{B}\right]$-code over $\mathbb{F}_{q}, \mathbb{F}_{q^{k_{B}}}$ an extension field of $\mathbb{F}_{q}$ with degree $k_{B}, \varphi$ an $\mathbb{F}_{q}$-linear isomorphism from $\mathbb{F}_{q^{k_{B}}}$ onto $B$ and $E$ a linear $\left[n_{E}, k_{E}, d_{E}\right]$-code over $\mathbb{F}_{q^{k_{B}}}$. The concatenated code of the inner code $B$ and the outer code $E$ is defined as

$$
B \square_{\varphi} E=\left\{\left(\varphi\left(c_{1}\right), \ldots, \varphi\left(c_{n_{E}}\right)\right) \mid\left(c_{1}, \ldots, c_{n_{E}}\right) \in E\right\}
$$

(cf. [?, Definition 2.1]). It is known that $B \square_{\varphi} E$ is a linear code over $\mathbb{F}_{q}$ with parameters $\left[n_{B} n_{E}, k_{B} k_{E}, \geq d_{B} d_{E}\right]$. 
From now on, let $m$ be a positive integer satisfying $\operatorname{gcd}(m, q)=1$ and $m \geq 3,\left(\mathbb{Z}_{m},+\right)$ the addition group of integer residue classes modulo $m$ where $\mathbb{Z}_{m}=\{0,1, \ldots, m-1\}$, and denote by

$$
\mathcal{A}=\mathbb{F}_{q}[x] /\left\langle x^{m}-1\right\rangle=\left\{\sum_{i=0}^{m-1} a_{i} x^{i} \mid a_{0}, a_{1}, \ldots, a_{m-1} \in \mathbb{F}_{q}\right\}
$$

the residue class ring of $\mathbb{F}_{q}[x]$ modulo its ideal generated by $x^{m}-1$ with operations defined by the usual polynomial operations modulo $x^{m}-1$. We will identify cyclic codes over $\mathbb{F}_{q}$ of length $m$ with ideals of the ring $\mathcal{A}$ under the identification map: $\left(a_{0}, a_{1}, \ldots, a_{m-1}\right) \mapsto \sum_{i=0}^{m-1} a_{i} x^{i}$.

First, we define

$$
\theta:\left(\mathbb{Z}_{m},+\right) \rightarrow\left(\mathbb{Z}_{m},+\right) \text { by } \theta(s) \equiv r s(\bmod m) .
$$

As $\operatorname{gcd}(m, r)=1$, we see that $\theta$ is a group automorphism on $\left(\mathbb{Z}_{m},+\right)$. Moreover, from $1<r<n$ and $r^{3} \equiv 1$ $(\bmod m)$ we deduce that the multiplicative order of $\theta$ is a factor of 3 .

Next, we define a map $\mathcal{A} \rightarrow \mathcal{A}$ by the rule that

$$
a(x) \mapsto \sum_{j=0}^{m-1} a_{j} x^{\theta(j)} \equiv a\left(x^{r}\right)\left(\bmod x^{m}-1\right),
$$

for any $a(x)=\sum_{j=0}^{m-1} a_{j} x^{j} \in \mathcal{A}$. In order to simplify notations, we also use $\theta$ to denote this map on $\mathcal{A}$, i.e.,

$$
\theta(a(x))=a\left(x^{r}\right) \text { in } \mathcal{A} .
$$

Then $\theta$ is an $\mathbb{F}_{q}$-algebra automorphism on $\mathcal{A}$ satisfying $\theta^{3}=\mathrm{id}_{\mathcal{A}}$. In addition, $\theta$ is a permutation of the coordinate positions $\{0,1, \ldots, m-1\}$ of a cyclic codes of length $m$ over $\mathbb{F}_{q}$ and is called a multiplier. Readers are referred to [?, Theorems 4.3.12 and 4.3.13] for more details on basic properties of multipliers.

Wether $\theta$ denotes this automorphism of $\mathcal{A}$ or the group automorphism on $\left(\mathbb{Z}_{m},+\right)$ is determined by the context. Let

$$
\mathcal{A}[y ; \theta]=\left\{\sum_{j=0}^{k} a_{j}(x) y^{j} \mid a_{0}(x), \ldots, a_{k}(x) \in \mathcal{A}, k \geq 0\right\}
$$

be the skew polynomial ring over the commutative $\operatorname{ring} \mathcal{A}$ with coefficients written on the left side, where the multiplication is defined by the rule

$$
y^{j} a(x)=\theta^{j}(a(x)) y^{j}=a\left(x^{r^{j}}\right) y^{j}, \forall a(x) \in \mathcal{A}
$$

and by the natural $\mathcal{A}$-linear extension to all polynomials in $\mathcal{A}[y ; \theta]$.

As $\theta^{3}=\operatorname{id}_{\mathcal{A}}$, we have $y^{3} a(x)=a(x) y^{3}$ for all $a(x) \in \mathcal{A}$. So $y^{3}-1$ generates a two-sided ideal $\left\langle y^{3}-1\right\rangle$ of $\mathcal{A}[y ; \theta]$. Let

$$
\mathcal{A}[y ; \theta] /\left\langle y^{3}-1\right\rangle=\left\{\alpha+\beta y+\gamma y^{2} \mid \alpha, \beta, \gamma \in \mathcal{A}\right\}
$$

be the residue class ring of $A[y ; \theta]$ modulo its two-sided ideal $\left\langle y^{3}-1\right\rangle$. For any $\xi=a_{0}(x)+a_{1}(x) y+a_{2}(x) y^{2} \in$ $\mathcal{A}[y ; \theta] /\left\langle y^{3}-1\right\rangle$, where $a_{j}(x)=\sum_{i=0}^{m-1} a_{i, j} x^{i} \in \mathcal{A}$ with $a_{i, j} \in \mathbb{F}_{q}$ for $j=0,1,2$, we define a natural map:

$$
\Phi: \xi \mapsto a(x, y)=\sum_{i=0}^{m-1} \sum_{j=0}^{2} a_{i, j} x^{i} y^{j} .
$$

Then it can be easily proved that $\Phi$ is a ring isomorphism from $\mathcal{A}[y ; \theta] /\left\langle y^{3}-1\right\rangle$ onto $\mathbb{F}_{q}\left[G_{(m, 3, r)}\right]$.

In the rest of this paper, we will identify $\mathbb{F}_{q}\left[G_{(m, 3, r)}\right]$ with $\mathcal{A}[y ; \theta] /\left\langle y^{3}-1\right\rangle$ under this ring isomorphism $\Phi$.

Theorem 2.1: Using the notations above, $\mathcal{C}$ is a left $G_{(m, 3, r)}$-code over $\mathbb{F}_{q}$ if and only if $\mathcal{C}$ is a left ideal of the ring $\mathcal{A}[y ; \theta] /\left\langle y^{3}-1\right\rangle$.

Proof: By the identification of $\mathbb{F}_{q}\left[G_{(m, 3, r)}\right]$ with $\mathcal{A}[y ; \theta] /\left\langle y^{3}-1\right\rangle$, we see that $\mathcal{C}$ is a left $G_{(m, 3, r)}$-code over $\mathbb{F}_{q}$, i.e., $\mathcal{C}$ is a left ideal of the ring $\mathbb{F}_{q}\left[G_{(m, 3, r)}\right]$, if and only if $\mathcal{C}$ is a left ideal of $\mathcal{A}[y ; \theta] /\left\langle y^{3}-1\right\rangle$. 
In order to determine all left $G_{(m, 3, r)}$-codes over $\mathbb{F}_{q}$, by Theorem 2.1 it is sufficient to give all left ideals of the ring $\mathcal{A}[y ; \theta] /\left\langle y^{3}-1\right\rangle$. To do this, we need investigate the structure and properties of $\mathcal{A}$ first.

For any integer $s, 0 \leq s \leq m-1$, let $J_{s}^{(q)}$ be the $q$-cyclotomic coset modulo $m$, i.e., $J_{s}^{(q)}=\left\{s, s q, \ldots, s q^{l_{s}-1}\right\}$ $(\bmod m)$ where $l_{s}=\min \left\{k \in \mathbb{Z}^{+} \mid s\left(q^{k}-1\right) \equiv 0(\bmod m)\right\}$. Then $\left|J_{s}^{(q)}\right|=l_{s}$. It is obvious that $J_{0}^{(q)}=\{0\}$ and $\theta\left(J_{0}^{(q)}\right)=J_{0}^{(q)}=\{0\}$. In this paper, we assume that

$$
r \equiv q^{\epsilon}(\bmod m)
$$

for some positive integer $\epsilon$.

Lemma 2.2: Let $0 \leq s \leq m-1$. Then $J_{s}^{(q)}$ satisfies one and only one of the following two conditions:

(I) $\theta(s) \equiv s(\bmod m)$. In this case, $\theta(k) \equiv k(\bmod m)$ for all $k \in J_{s}^{(q)}$.

(II) $\theta(s) \in J_{s}^{(q)}$ and $\theta(s) \not \equiv s(\bmod m)$. In this case, $l_{s}=\left|J_{s}^{(q)}\right|$ is a multiple of 3 , and $\theta\left(J_{s}^{(q)}\right)=J_{s}^{(q)}$.

Proof: By Condition (1), it follows that $\theta(s) \in J_{s}^{(q)}$ and $\theta\left(J_{s}^{(q)}\right)=J_{s}^{(q)}$. Then we have one of the following two cases.

(I) $\theta(s)=r s \equiv s(\bmod m)$. In this case, for any $k \in J_{s}^{(q)}$ we have $k \equiv s q^{j}$ for some $0 \leq j \leq l_{s}-1$, and hence $\theta(k)=r s q^{j} \equiv s q^{j} \equiv k(\bmod m)$.

(II) $\theta(s) \in J_{s}^{(q)}$ and $\theta(s) \not \equiv s(\bmod m)$. In this case, it is obvious that $l_{s}=\left|J_{s}^{(q)}\right| \geq 2$. By $\theta(s) \in J_{s}^{(q)}$, there exits integer $v, 1 \leq v \leq l_{s}-1$, such that $\theta(s)=r s \equiv s q^{v}(\bmod m)$. By $r^{3} \equiv 1(\bmod m)$ and $s q^{v} \equiv r s(\bmod m)$, we have $s q^{3 v}=s\left(q^{v}\right)^{3} \equiv r^{3} s \equiv s(\bmod m)$. From this and by the minimality of $l_{s}$, we deduce that $l_{s} \mid 3 v$. Suppose that $l_{s}$ is not a multiple of 3 . Then $\operatorname{gcd}\left(l_{s}, 3\right)=1$ and hence $l_{s} \mid v$. By $s q^{l_{s}} \equiv s(\bmod m)$, we deduce that $s q^{v} \equiv s$ $(\bmod m)$, i.e., $\theta(s) \equiv s(\bmod m)$, which contradicts that $\theta(s) \not \equiv s(\bmod m)$. Hence $l_{s}$ is a multiple of 3 .

In this paper, let $\zeta$ be a primitive $m$ th root of unity in an extension field of $\mathbb{F}_{q}$. Then $x^{m}-1=\sum_{s=0}^{m-1}\left(x-\zeta^{s}\right)$. We will adopt the following notations:

- Let $J_{k_{0}}^{(q)}, J_{k_{1}}^{(q)}, \ldots, J_{k_{s}}^{(q)}$, where $k_{0}=0$, be all distinct $q$-cyclotomic cosets modulo $m$ satisfying Condition (I) in Lemma 2.2.

- Let $J_{k_{s+1}}^{(q)}, \ldots, J_{k_{s+t}}^{(q)}$ be all distinct $q$-cyclotomic cosets modulo $m$ satisfying Condition (II) in Lemma 2.2

- Denote $J(i)=J_{k_{i}}^{(q)}, f_{i}(x)=\prod_{j \in J(i)}\left(x-\zeta^{j}\right)$,

$$
K_{i}=\mathbb{F}_{q}[x] /\left\langle f_{i}(x)\right\rangle
$$

and assume $d_{i}=\operatorname{deg}\left(f_{i}(x)\right)=\left|J_{k_{i}}^{(q)}\right|=|J(i)|$ for all $i=0,1, \ldots, s+t$.

Then $d_{i}$ is a multiple of 3 for all $s+1 \leq i \leq s+t$ by Lemma 2.2(II). It is clear that $f_{0}(x), f_{1}(x), \ldots, f_{s+t}(x)$ are pairwise coprime irreducible polynomials in $\mathbb{F}_{q}[x]$ satisfying

$$
x^{m}-1=f_{0}(x) f_{1}(x) \ldots f_{s+t}(x) .
$$

Hence $K_{i}$ is an extension field of $\mathbb{F}_{q}$ with cardinality $q^{d_{i}}$ for $i=0,1, \ldots, s+t$, and $m=\sum_{i=0}^{s+t} d_{i}$.

For each integer $i, 0 \leq i \leq s+t$, denote

$$
F_{i}(x)=\frac{x^{m}-1}{f_{i}(x)} \in \mathbb{F}_{q}[x] .
$$

Then $\operatorname{gcd}\left(F_{i}(x), f_{i}(x)\right)=1$. By Extended Euclidian Algorithm, we find polynomials $u_{i}(x), v_{i}(x) \in \mathbb{F}_{q}[x]$ such that

$$
u_{i}(x) F_{i}(x)+v_{i}(x) f_{i}(x)=1
$$

In this paper, we denote

$$
\varepsilon_{i}(x) \equiv u_{i}(x) F_{i}(x)=1-v_{i}(x) f_{i}(x)\left(\bmod x^{m}-1\right) .
$$

By (2) and (3), it follows that $\varepsilon_{i}\left(\zeta^{j}\right)=1$ for all $j \in J(i)$ and $\varepsilon_{i}\left(\zeta^{j}\right)=0$ for all $j \in \mathbb{Z}_{m} \backslash J(i)$, which implies

$$
\varepsilon_{i}(x)=\frac{1}{m} \sum_{l=0}^{m-1}\left(\sum_{j \in J(i)} \zeta^{-j l}\right) x^{l}, 0 \leq i \leq s+t .
$$


Then we have the following conclusions.

Lemma 2.3: Using the notations above, the following hold.

(i) $\sum_{i=0}^{s+t} \varepsilon_{i}(x)=1, \varepsilon_{i}(x)^{2}=\varepsilon_{i}(x)$ and $\varepsilon_{i}(x) \varepsilon_{j}(x)=0$ in $\mathcal{A}$ for all $0 \leq i \neq j \leq s+t$.

(ii) $\mathcal{A}=\mathcal{A}_{0} \oplus \mathcal{A}_{1} \oplus \ldots \mathcal{A}_{s+t}$, where $\mathcal{A}_{i}=\mathcal{A} \varepsilon_{i}(x)$ is the ideal of $\mathcal{A}$ generated by $\varepsilon_{i}(x)$, and $\mathcal{A}_{i}$ is a commutative ring with $\varepsilon_{i}(x)$ as its multiplicative identity for all $i=0,1, \ldots, s+t$.

(iii) For each integer $i, 0 \leq i \leq s+t$, define

$$
\varphi_{i}: a(x) \mapsto \varepsilon_{i}(x) a(x) \bmod x^{m}-1\left(\forall a(x) \in K_{i}\right)
$$

Then $\varphi_{i}$ is a field isomorphism from $K_{i}$ onto $\mathcal{A}_{i}$.

(iv) $\theta\left(\varepsilon_{i}(x)\right)=\varepsilon_{i}(x)$ and $\theta\left(\mathcal{A}_{i}\right)=\mathcal{A}_{i}$, for all $i=0,1, \ldots, s+t$.

(v) For each integer $i, 0 \leq i \leq s+t$, define

$$
\theta_{i}: K_{i} \rightarrow K_{i} \text { via } a(x) \mapsto a\left(x^{r}\right)\left(\forall a(x) \in K_{i}\right) .
$$

Then $\theta_{i}$ is an $\mathbb{F}_{q}$-algebra automorphism on $K_{i}$ satisfying $\theta_{i}^{3}=\operatorname{id}_{K_{i}}$, where $\operatorname{id}_{K_{i}}$ is the identity automorphism on $K_{i}$. Precisely, $\theta_{i}=\operatorname{id}_{K_{i}}$ for all $i=0,1, \ldots, s$, and the multiplicative order of $\theta_{i}$ is equal to 3 for all $i=s+1, \ldots, s+t$.

(vi) The restriction $\left.\theta\right|_{\mathcal{A}_{i}}$ of $\theta$ on $\mathcal{A}_{i}$ is an $\mathbb{F}_{q}$-algebra automorphism on $\mathcal{A}_{i}$ satisfying $\left(\left.\theta\right|_{\mathcal{A}_{i}}\right)^{3}=\mathrm{id}_{\mathcal{A}_{i}}$ and $\left.\theta\right|_{\mathcal{A}_{i}}=\varphi_{i} \theta_{i} \varphi_{i}^{-1}$. Hence the following diagram for $\mathbb{F}_{q}$-algebra isomorphisms commutes:

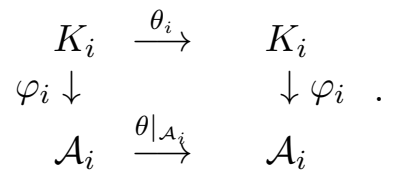

Then $\left.\theta\right|_{\mathcal{A}_{i}}=\operatorname{id}_{\mathcal{A}_{i}}$ for all $i=0,1, \ldots, s$, and the multiplicative order of $\left.\theta\right|_{\mathcal{A}_{i}}$ is equal to 3 for all $i=s+1, \ldots, s+t$.

Proof: (i)-(iii) follow from classical ring theory and Equations (2) and (3).

(iv) By the definition of the automorphism $\theta$ on $\mathcal{A}$ and (4), it follows that

$$
\theta\left(\varepsilon_{i}(x)\right)=\frac{1}{m} \sum_{l=0}^{m-1}\left(\sum_{j \in J(i)} \zeta^{-j l}\right) x^{r l(\bmod m)}
$$

As $J(i)=\theta(J(i))=\{r j(\bmod m) \mid j \in J(i)\}$, we have

$$
\begin{aligned}
\theta\left(\varepsilon_{i}(x)\right) & =\frac{1}{m} \sum_{l=0}^{m-1}\left(\sum_{j \in J(i)} \zeta^{-(r j) l}\right) x^{r l(\bmod m)} \\
& =\frac{1}{m} \sum_{l=0}^{m-1}\left(\sum_{j \in J(i)} \zeta^{-j(r l)}\right) x^{r l(\bmod m)} .
\end{aligned}
$$

Moreover, since $\theta$ is an automorphism of the group $\left(\mathbb{Z}_{m},+\right)$, for each $k \in \mathbb{Z}_{m}$ there is a unique $l \in \mathbb{Z}_{m}$ such that $k=r l(\bmod m)$, and hence

$$
\theta\left(\varepsilon_{i}(x)\right)=\frac{1}{m} \sum_{k=0}^{m-1}\left(\sum_{j \in J(i)} \zeta^{-j k}\right) x^{k}=\varepsilon_{i}(x) .
$$

Therefore, $\theta\left(\mathcal{A}_{i}\right)=\theta(\mathcal{A}) \theta\left(\varepsilon_{i}(x)\right)=\mathcal{A} \varepsilon_{i}(x)=\mathcal{A}_{i}$.

(v) It is clear that $\theta_{i}$ is an $\mathbb{F}_{q}$-algebra endomorphism of $K_{i}$. By $r^{3} \equiv 1(\bmod m)$ and $f_{i}(x)=\prod_{j \in J(i)}\left(x-\zeta^{j}\right)$, we see that $\left(\zeta^{j}\right)^{r^{3}}=\zeta^{j}$ for all $j \in J(i)$, which implies that $x^{r^{3}} \equiv x\left(\bmod f_{i}(x)\right)$, i.e., $x^{r^{3}}=x$ in $K_{i}$. Hence for any $a(x) \in K_{i}=\mathbb{F}_{q}[x] /\left\langle f_{i}(x)\right\rangle$ we have $\theta_{i}^{3}(a(x))=a\left(x^{r^{3}}\right)=a(x)$ in $K_{i}$. Therefore, $\theta_{i}^{3}=\operatorname{id}_{K_{i}}$ and so $\theta_{i}$ is an $\mathbb{F}_{q}$-algebra automorphism of $K_{i}$. Then we consider the following two cases:

(v-1) Let $0 \leq i \leq s$. For any $j \in J(i)$, by Condition (I) in Lemma 2.2 we have $\left(\zeta^{j}\right)^{r}=\zeta^{j}$, which implies $x^{r} \equiv x\left(\bmod x-\zeta^{j}\right)$, and hence $x^{r} \equiv x\left(\bmod f_{i}(x)\right)$. Therefore, $\theta_{i}(a(x))=a\left(x^{r}\right)=a(x)$ for any $a(x) \in K_{i}=$ $\mathbb{F}_{q}[x] /\left\langle f_{i}(x)\right\rangle$, and so $\theta_{i}=\mathrm{id}_{K_{i}}$. 
(v-2) Let $s+1 \leq i \leq s+t$. By Condition (II) in Lemma 2.2, there exists $j \in J(i)$ such that $r j \not \equiv j(\bmod m)$, which implies $\left(\zeta^{j}\right)^{r} \neq \zeta^{j}$. Then by the proof of (v-1), we conclude $\theta_{i} \neq \mathrm{id}_{K_{i}}$. From this and by $\theta_{i}^{3}=\mathrm{id}_{K_{i}}$, we deduce that the multiplicative order of $\theta_{i}$ is equal to 3 .

(vi) By (iv) and $\theta^{3}=\mathrm{id}_{\mathcal{A}}$, it follows that $\left.\theta\right|_{\mathcal{A}_{i}}$ is an $\mathbb{F}_{q}$-algebra automorphism on $\mathcal{A}_{i}$ satisfying $\left(\left.\theta\right|_{\mathcal{A}_{i}}\right)^{3}=\mathrm{id}_{\mathcal{A}_{i}}$. Then the equation $\left.\theta\right|_{\mathcal{A}_{i}}=\varphi_{i} \theta_{i} \varphi_{i}^{-1}$ follows from (v) and the definitions of $\varphi_{i}, \theta_{i}$ and $\left.\theta\right|_{\mathcal{A}_{i}}$ immediately.

For any integer $i, 0 \leq i \leq s+t$, it is known that $\mathcal{A}_{i}$ is a minimal cyclic code of length $m$ over $\mathbb{F}_{q}$. Precisely, $f_{i}(x)$ is the parity check polynomial and $\varepsilon_{i}(x)$ is the idempotent generator of $\mathcal{A}_{i}$. Hence $\operatorname{dim}_{\mathbb{F}_{q}}\left(\mathcal{A}_{i}\right)=\operatorname{deg}\left(f_{i}(x)\right)=d_{i}$.

Frow now on, we adopt the following notations.

- Let $K_{i}\left[y ; \theta_{i}\right]=\left\{\sum_{j=0}^{k} b_{j}(x) y^{j} \mid b_{0}(x), \ldots, b_{k}(x) \in K_{i}, k \geq 0\right\}$ be the skew polynomial ring over $K_{i}$ with coefficients written on the left side, where the multiplication is defined by the rule

$$
y^{j} a(x)=\theta_{i}^{j}(a(x)) y^{j}=a\left(x^{r^{j}}\right) y^{j}, \forall a(x) \in K_{i}
$$

and by the natural $K_{i}$-linear extension to all polynomials in $K_{i}\left[y ; \theta_{i}\right]$.

Since $\theta_{i}^{3}=\operatorname{id}_{K_{i}}$ by Lemma $2.3(\mathrm{v})$, we see that $y^{3}-1$ generates a two-sided ideal $\left\langle y^{3}-1\right\rangle$ of $K_{i}\left[y ; \theta_{i}\right]$.

- Let $K_{i}\left[y ; \theta_{i}\right] /\left\langle y^{3}-1\right\rangle=\left\{a(x)+b(x) y+c(x) y^{2} \mid a(x), b(x), c(x) \in K_{i}\right\}$ be the residue class ring of $K_{i}\left[y ; \theta_{i}\right]$ modulo its two-sided ideal $\left\langle y^{3}-1\right\rangle$.

Recall that left ideals of $K_{i}\left[y ; \theta_{i}\right] /\left\langle y^{3}-1\right\rangle$ are called skew $\theta_{i}$-cyclic codes over $K_{i}$ of length 3 (See [?, Theorem 1]). For more details on skew cyclic codes, readers are referred to [?], [?], [?], [?], [?].

Now, we can decompose any left $G_{(m, 3, r)}$-code into a direct sum of concatenated codes by the following theorem.

Theorem 2.4: Using the notations above, we have the following conclusions.

(i) $\mathcal{A}[y ; \theta] /\left\langle y^{3}-1\right\rangle=\oplus_{i=0}^{s+t}\left(\mathcal{A}_{i}\left[y ;\left.\theta\right|_{\mathcal{A}_{i}}\right] /\left\langle\varepsilon_{i}(x) y^{3}-\varepsilon_{i}(x)\right\rangle\right)$.

(ii) For each integer $i, 0 \leq i \leq s+t, \varphi_{i}: K_{i} \rightarrow \mathcal{A}_{i}$ can be extended to a ring isomorphism from $K_{i}\left[y ; \theta_{i}\right] /\left\langle y^{3}-1\right\rangle$ onto $\mathcal{A}_{i}\left[y ;\left.\theta\right|_{\mathcal{A}_{i}}\right] /\left\langle\varepsilon_{i}(x) y^{3}-\varepsilon_{i}(x)\right\rangle$ by

$$
\varphi_{i}: \xi(y) \mapsto \varphi_{i}\left(\xi_{0}\right)+\varphi_{i}\left(\xi_{1}\right) y+\varphi_{i}\left(\xi_{2}\right) y^{2}=\varepsilon_{i}(x) \xi(y)
$$

for any $\xi(y)=\xi_{0}+\xi_{1}+\xi_{2} y^{2}$ with $\xi_{0}, \xi_{1}, \xi_{2} \in K_{i}$.

(iii) $\mathcal{C}$ is a left $G_{(m, 3, r)}$-code over $\mathbb{F}_{q}$ if and only if for each $0 \leq i \leq s+t$ there is a unique skew $\theta_{i}$-cyclic code $C_{i}$ over $K_{i}$ of length 3 such that

$$
\mathcal{C}=\bigoplus_{i=0}^{s+t} \mathcal{A}_{i} \square_{\varphi_{i}} C_{i}
$$

where $\mathcal{A}_{i} \square_{\varphi_{i}} C_{i}=\left\{\varepsilon_{i}(x) \xi\left(\bmod x^{m}-1\right) \mid \xi \in C_{i}\right\}$. Moreover, we have $|\mathcal{C}|=\prod_{i=0}^{s+t}\left|C_{i}\right|$.

Proof: (i) By Lemma 2.3(ii),(iv) and (vi), we have

$$
\mathcal{A}[y ; \theta]=\oplus_{i=0}^{s+t} \mathcal{A}_{i}\left[y ;\left.\theta\right|_{\mathcal{A}_{i}}\right] .
$$

Moreover, by $\mathcal{A}_{i}=\mathcal{A} \varepsilon_{i}(x)$ we know that the projection from $\mathcal{A}[y ; \theta]$ onto $\mathcal{A}_{i}\left[y ;\left.\theta\right|_{\mathcal{A}_{i}}\right]$ is determined by $\alpha(y) \mapsto$ $\varepsilon_{i}(x) \alpha(y)(\forall \alpha(y) \in \mathcal{A}[y ; \theta])$. Especially, we have $y^{3}-1 \mapsto \varepsilon_{i}(x) y^{3}-\varepsilon_{i}(x)$ under this projection. As $\left(\left.\theta\right|_{\mathcal{A}_{i}}\right)^{3}=\mathrm{id}_{\mathcal{A}_{i}}$, $\varepsilon_{i}(x) y^{3}-\varepsilon_{i}(x)$ generates a two-sided ideal $\left\langle\varepsilon_{i}(x) y^{3}-\varepsilon_{i}(x)\right\rangle$ of $\mathcal{A}_{i}\left[y ; \theta \mid{ }_{\mathcal{A}_{i}}\right]$ for all $i=0,1, \ldots, s+t$. By Lemma2.3 (i) it follows that $y^{3}-1=\sum_{i=0}^{s+t}\left(\varepsilon_{i}(x) y^{3}-\varepsilon_{i}(x)\right)$ and $\left(\varepsilon_{i}(x) y^{3}-\varepsilon_{i}(x)\right)\left(\varepsilon_{j}(x) y^{3}-\varepsilon_{j}(x)\right)=0$ for all $0 \leq i \neq j \leq s+t$. Hence

$$
\mathcal{A}[y ; \theta] /\left\langle y^{3}-1\right\rangle=\oplus_{i=0}^{s+t}\left(\mathcal{A}_{i}\left[y ;\left.\theta\right|_{\mathcal{A}_{i}}\right] /\left\langle\varepsilon_{i}(x) y^{3}-\varepsilon_{i}(x)\right\rangle\right) .
$$

(ii) Since $\varphi_{i}: K_{i} \rightarrow \mathcal{A}_{i}$ is a ring isomorphism by Lemma 2.3(iii), the conclusion follows from Lemma 2.3 (vi) and a direct calculation.

(iii) By Theorem 2.1 and (i), we see that $\mathcal{C}$ is a left $G_{(m, 3, r)}$-code over $\mathbb{F}_{q}$ if and only if for each integer $i$, $0 \leq i \leq s+t$, there is a unique left ideal $\mathcal{C}_{i}$ of the ring $\mathcal{A}_{i}\left[y ;\left.\theta\right|_{\mathcal{A}_{i}}\right] /\left\langle\varepsilon_{i}(x) y^{3}-\varepsilon_{i}(x)\right\rangle$ such that $\mathcal{C}=\oplus_{i=0}^{s+t} \mathcal{C}_{i}$. By (ii), the latter condition is equivalent to that for each integer $i, 0 \leq i \leq s+t$, there is a unique left ideal $C_{i}$ of $K_{i}\left[y ; \theta_{i}\right] /\left\langle y^{3}-1\right\rangle$ such that $\mathcal{C}_{i}=\varphi_{i}\left(C_{i}\right)=\left\{\varepsilon_{i}(x) \xi \mid \xi \in C_{i}\right\}=\mathcal{A}_{i} \square_{\varphi_{i}} \bar{C}_{i}$.

Finally, it is clear that the codewords contained in $\mathcal{C}$ is equal to $|\mathcal{C}|=\prod_{i=0}^{s+t}\left|\mathcal{C}_{i}\right|=\prod_{i=0}^{s+t}\left|C_{i}\right|$. 
By Theorem 2.4, in order to give all distinct left $G_{(m, 3, r)}$-codes over $\mathbb{F}_{q}$ it is sufficient to determine all distinct skew $\theta_{i}$-cyclic codes over $K_{i}$ of length 3 , for all $i=0,1, \ldots, s+t$. For convenience and notations simplicity in the following sections, we introduce the following notations.

Notation 2.5: For any integer $i, 0 \leq i \leq s+t$, denote

- $\mathcal{R}_{i}=K_{i}\left[y ; \theta_{i}\right] /\left\langle y^{3}-1\right\rangle$.

For any $\alpha=a_{0}+a_{1} y+a_{2} y^{2} \in \mathcal{R}_{i}$ with $a_{0}, a_{1}, a_{2} \in K_{i}$, the Hamming weight $\mathrm{wt}_{H}^{(i)}(\alpha)$ of $\alpha$ over $K_{i}$ is defined as the number of nonzero coefficients of the polynomial $a_{0}+a_{1} y+a_{2} y^{2} \in K_{i}\left[y\right.$; $\left.\theta_{i}\right]$, i.e.,

- $\mathrm{wt}_{H}^{(i)}(\alpha)=\left|\left\{j \mid a_{j} \neq 0, j=0,1,2\right\}\right|$.

For any nonzero left ideal $J$ of $\mathcal{R}_{i}$, the minimum Hamming weight $\mathrm{wt}_{H}^{(i)}(J)$ of $J$ over $K_{i}$ is defined as

- $\mathrm{wt}_{H}^{(i)}(J)=\min \left\{\mathrm{wt}_{H}^{(i)}(\alpha) \mid \alpha \neq 0, \alpha \in J\right\}$.

\section{SKEW $\theta_{i}$-CYCLIC CODES OVER $K_{i}$ OF LENGTH 3}

In this section, we give all skew $\theta_{i}$-cyclic codes over $K_{i}$ of length 3 , i.e., left ideals of the ring $\mathcal{R}_{i}$, where $0 \leq i \leq s+t$.

$\diamond$ Let $0 \leq i \leq s$. Then $K_{i}$ is a finite field of cardinality $q^{d_{i}}$ and $\theta_{i}$ is the identity automorphism of $K_{i}$ by Lemma 2.3 v). Hence $\mathcal{R}_{i}=K_{i}[y] /\left\langle y^{3}-1\right\rangle$ which is a commutative ring. In this case, left ideals of $\mathcal{R}_{i}$ are in fact ideals of $\mathcal{R}_{i}$. By the basic theory of cyclic codes over finite fields, we know that $C_{i}$ is an ideal of $\mathcal{R}_{i}$ if and only if $C_{i}$ is a cyclic code over $K_{i}$ of length 3 . The latter is equivalent to that there is a unique monic divisor $g(y)$ of $y^{3}-1$ in $K_{i}[y]$ such that $C_{i}=\mathcal{R}_{i} g(y)$. Then $g(y)$ is called the generator polynomial of $C_{i}$ and $\operatorname{dim}_{K_{i}}\left(C_{i}\right)=3-\operatorname{deg}_{y}(g(y))$ where $\operatorname{deg}_{y}(g(y))$ is the degree of $g(y)$ as a polynomial with indeterminate $y$. Obviously, $\mathcal{R}_{i}\left(y^{3}-1\right)=\{0\}$.

Theorem 3.1: Let $0 \leq i \leq s$. Then $\mathcal{R}_{i}=K_{i}[y] /\left\langle y^{3}-1\right\rangle$ which is a commutative ring, and the following hold. (i) If $q \equiv 0(\bmod 3)$, there are 4 distinct ideals in $\mathcal{R}_{i}$ :

$$
\mathcal{R}_{i} g(y), \text { where } g(y) \in\left\{1, y-1,(y-1)^{2}, y^{3}-1\right\} .
$$

(ii) If $q^{d_{i}} \equiv 2(\bmod 3)$, there are 4 distinct ideals in $\mathcal{R}_{i}$ :

$$
\mathcal{R}_{i} g(y) \text {, where } g(y) \in\left\{1, y-1, y^{2}+y+1, y^{3}-1\right\} .
$$

(iii) Let $q^{d_{i}} \equiv 1(\bmod 3), \zeta_{i}(x)$ a primitive element of $K_{i}$ and denote $\omega_{i}(x)=\zeta_{i}(x)^{\frac{q^{d_{i}-1}}{3}}\left(\bmod f_{i}(x)\right)$. Then there are 8 distinct ideals in $\mathcal{R}_{i}: \mathcal{R}_{i} g(y)$, where

$$
\begin{aligned}
& g(y) \in\left\{1, y-1, y-\omega_{i}(x), y-\omega_{i}(x)^{2}, y^{2}+y+1,\right. \\
& \left.\quad(y-1)\left(y-\omega_{i}(x)^{2}\right),(y-1)\left(y-\omega_{i}(x)\right), y^{3}-1\right\} .
\end{aligned}
$$

Proof: (i) Since $q \equiv 0(\bmod 3)$, we have $y^{3}-1=(y-1)^{3}$ in $K_{i}[y]$. In this case, $y^{3}-1$ has 4 monic divisors in $K_{i}[y]: 1, y-1,(y-1)^{2}$ and $y^{3}-1$,.

(ii) Since $q^{d_{i}} \equiv 2(\bmod 3)$, we have that $y^{3}-1=(y-1)\left(y^{2}+y+1\right)$ where $y^{2}+y+1$ is irreducible in $K_{i}[y]$. In this case, $y^{3}-1$ has 4 monic divisors in $K_{i}[y]: 1, y-1, y^{2}+y+1$ and $y^{3}-1$.

(iii) Since $q^{d_{i}} \equiv 1(\bmod 3)$, we have $3 \mid\left(q^{d_{i}}-1\right)$ and $\omega_{i}(x)$ is a primitive 3 th root of unity in $K_{i}$, which implies $y^{3}-1=(y-1)\left(y-\omega_{i}(x)\right)\left(y-\omega_{i}(x)^{2}\right)$. In this case, $y^{3}-1$ has 8 monic divisors in $K_{i}[y]: 1, y-1, y-\omega_{i}(x)$, $y-\omega_{i}(x)^{2}, y^{2}+y+1,(y-1)\left(y-\omega_{i}(x)^{2}\right),(y-1)\left(y-\omega_{i}(x)\right)$ and $y^{3}-1$.

$\diamond$ Let $s+1 \leq i \leq s+t$. Then $K_{i}$ is a finite field of cardinality $q^{d_{i}}, d_{i}$ is a multiple of 3 by Lemma 2.2(II), and $\theta_{i}$ is an $\mathbb{F}_{q}$-algebra automorphism of $K_{i}$ with multiplicative order 3 by Lemma 2.3 (v).

In this case, by [?, Lemma 1 and Theorem 1] we know that $C_{i}$ is a left ideal of $\mathcal{R}_{i}$ if and only if $C_{i}$ is a skew $\theta_{i}$-cyclic code over $K_{i}$ of length 3 , and the latter is equivalent to that there is a unique monic right divisor $g(y)$ of $y^{3}-1$ in the skew polynomial ring $K_{i}\left[y ; \theta_{i}\right]$ such that $C_{i}=\mathcal{R}_{i} g(y)$. If the latter condition is satisfied, $g(y)$ is called the generator polynomial of $C_{i}, \operatorname{dim}_{K_{i}}\left(C_{i}\right)=3-\operatorname{deg}_{y}(g(y))$ and the number of 
codewords in $C_{i}$ is equal to $\left|C_{i}\right|=\left(q^{d_{i}}\right)^{3-\operatorname{deg}_{y}(g(y))}$. Precisely, a generator matrix of $C_{i}$ over $K_{i}$ is given by: $G_{C_{i}}=(a(x), b(x), 1)$ if $g(x)=a(x)+b(x) y+y^{2} ;$ and

$$
G_{C_{i}}=\left(\begin{array}{ccc}
a(x) & 1 & 0 \\
0 & \theta_{i}(a(x)) & 1
\end{array}\right) \text { if } g(x)=a(x)+y .
$$

In order to describe monic right divisors of $y^{3}-1$ in the skew polynomial ring $K_{i}\left[y ; \theta_{i}\right]$ and the relationships between two nontrivial monic right divisors, we adopt the following notations in the rest of this paper:

- Let $\zeta_{i}(x)$ be a primitive element of $K_{i}$ and denote $\varrho_{i}(x)=\zeta_{i}(x)^{q^{\frac{d_{i}}{3}-1}}$. Then the multiplicative order of $\varrho_{i}(x)$ is equal to $\operatorname{ord}\left(\varrho_{i}(x)\right)=\frac{q^{d_{i}}-1}{q^{\frac{d_{i}}{3}}-1}=1+q^{\frac{d_{i}}{3}}+q^{\frac{2 d_{i}}{3}}$. Denote

$$
\mathcal{G}_{i}=\left\{\varrho_{i}(x)^{k} \mid k=0,1, \ldots, q^{\frac{d_{i}}{3}}+q^{\frac{2 d_{i}}{3}}\right\}
$$

which is the multiplicative cyclic subgroup of $K_{i}^{\times}$generated by $\varrho_{i}(x)$. Then $\left|\mathcal{G}_{i}\right|=1+q^{\frac{d_{i}}{3}}+q^{\frac{2 d_{i}}{3}}$.

Lemma 3.2: Let $s+1 \leq i \leq s+t$. Then we have the following:

(i) $\theta_{i}(a(x))=a(x)^{q^{\frac{d_{i}}{3}}}$ for all $a(x) \in K_{i}$ or $\theta_{i}(a(x))=a(x)^{q^{\frac{2 d_{i}}{3}}}$ for all $a(x) \in K_{i}$.

(ii) For any $\alpha(x) \in K_{i}^{\times}, \alpha(x) \in \mathcal{G}_{i}$ if and only if $\alpha(x)$ satisfies the following equation

$$
\alpha(x) \theta_{i}(\alpha(x)) \theta_{i}^{2}(\alpha(x))=1 .
$$

(iii) All distinct monic right divisors of $y^{3}-1$ with degree 1 in the skew polynomial ring $K_{i}\left[y ; \theta_{i}\right]$ are given by:

$$
-\alpha(x)+y, \alpha(x) \in \mathcal{G}_{i} .
$$

(iv) All distinct monic right divisors of $y^{3}-1$ with degree 2 in the skew polynomial ring $K_{i}\left[y ; \theta_{i}\right]$ are given by:

$$
\alpha(x)^{-1}+\theta_{i}^{2}(\alpha(x)) y+y^{2}, \alpha(x) \in \mathcal{G}_{i} .
$$

(v) For any $\alpha(x) \in \mathcal{G}_{i}$, we have

$$
\begin{aligned}
y^{3}-1 & =(-\alpha(x)+y) \cdot\left(\alpha(x)^{-1}+\theta_{i}^{2}(\alpha(x)) y+y^{2}\right) \\
& =\left(\alpha(x)^{-1}+\theta_{i}^{2}(\alpha(x)) y+y^{2}\right) \cdot(-\alpha(x)+y) .
\end{aligned}
$$

Therefore, both the number of right divisors of $y^{3}-1$ in $K_{i}\left[y ; \theta_{i}\right]$ with degree 1 and the number of right divisors of $y^{3}-1$ in $K_{i}\left[y ; \theta_{i}\right]$ with degree 2 are equal to $1+q^{\frac{d_{i}}{3}}+q^{\frac{2 d_{i}}{3}}$.

Proof: (i) Let $\sigma: \alpha \mapsto \alpha^{q}\left(\forall \alpha \in K_{i}\right)$ be the Frobenius automorphism of $K_{i}$ over $\mathbb{F}_{q}$. Then the multiplicative order of $\sigma$ is $d_{i}$ and every automorphism of $K_{i}$ over $\mathbb{F}_{q}$ is of the form: $\sigma^{k}, 0 \leq k \leq d_{i}-1$. By Lemma 2.2, $d_{i}$ is a multiple of 3 . Hence $\operatorname{ord}\left(\sigma^{k}\right)=3$ if and only if $\operatorname{gcd}\left(k, d_{i}\right)=\frac{d_{i}}{3}$, i.e., $k=\frac{\overline{d_{i}}}{3}$ or $k=\frac{2 d_{i}}{3}$. By Lemma 2.3 $(\mathrm{v}), \theta_{i}$ is an automorphism of $K_{i}$ over $\mathbb{F}_{q}$ with multiplicative order 3 , which implies that $\theta_{i}=\sigma^{\frac{d_{i}}{3}}$ or $\theta_{i}=\sigma^{\frac{2 d_{i}}{3}}$. Hence $\theta_{i}(a(x))=a(x)^{q^{\frac{d_{i}}{3}}}$ for all $a(x) \in K_{i}$ or $\theta_{i}(a(x))=a(x)^{\frac{2 d_{i}}{3}}$ for all $a(x) \in K_{i}$.

(ii) Denote $\alpha=\alpha(x)$ in order to simplify the notation. When $\theta_{i}(a(x))=a(x)^{q^{\frac{d_{i}}{3}}}$ for all $a(x) \in K_{i}$, it is clear that

$$
\alpha \theta_{i}(\alpha) \theta_{i}^{2}(\alpha)=\alpha^{1+q^{\frac{d_{i}}{3}}+q^{\frac{2 d_{i}}{3}}} .
$$

When $\theta_{i}(a(x))=a(x)^{q^{\frac{2 d_{i}}{3}}}$ for all $a(x) \in K_{i}$, by $\alpha^{q^{\frac{4 d_{i}}{3}}}=\left(\alpha^{q^{d_{i}}}\right)^{q^{\frac{d_{i}}{3}}}=\alpha^{q^{\frac{d_{i}}{3}}}$ we have $\alpha \theta_{i}(\alpha) \theta_{i}^{2}(\alpha)=\alpha^{1+q^{\frac{2 d_{i}}{3}}+q^{\frac{4 d_{i}}{3}}}=$ $\alpha^{1+q^{\frac{d_{i}}{3}}+q^{\frac{2 d_{i}}{3}}}$ as well.

Since $K_{i}^{\times}$is a multiplicative cyclic group with order $q^{d_{i}}-1$ and $\mathcal{G}_{i}$ is a subgroup of $K_{i}^{\times}$with order $1+q^{\frac{d_{i}}{3}}+q^{\frac{2 d_{i}}{3}}$, by basic group theory we conclude that $\alpha \in \mathcal{G}_{i}$ if and only if $\alpha^{1+q^{\frac{d_{i}}{3}}+q^{\frac{2 d_{i}}{3}}}=1$, i.e., $\alpha \theta_{i}(\alpha) \theta_{i}^{2}(\alpha)=1$.

(iii) Let $\alpha \in K_{i}$. Dividing $y^{3}-1$ by $y-\alpha$ from right hand in the skew polynomial ring $K_{i}\left[y ; \theta_{i}\right]$, we

$$
\begin{aligned}
y^{3}-1= & \left(y^{2}+\theta_{i}^{2}(\alpha) y+\theta_{i}^{2}(\alpha) \theta_{i}(\alpha)\right)(y-\alpha) \\
& +1-\alpha \theta_{i}(\alpha) \theta_{i}^{2}(\alpha) .
\end{aligned}
$$

Hence $-\alpha+y$ is a right divisors of $y^{3}-1$ in $K_{i}\left[y ; \theta_{i}\right]$ if and only if $1-\alpha \theta_{i}(\alpha) \theta_{i}^{2}(\alpha)=0$, i.e., $\alpha \theta_{i}(\alpha) \theta_{i}^{2}(\alpha)=1$. 
(iv) Let $\beta, \gamma \in K_{i}$. Then $\gamma+\beta y+y^{2}$ is a right divisors of $y^{3}-1$ in $K_{i}\left[y ; \theta_{i}\right]$ if and only if there exists $\alpha \in K_{i}$ such that

$$
\begin{aligned}
y^{3}-1 & =(-\alpha+y)\left(\gamma+\beta y+y^{2}\right) \\
& =y^{3}+\left(\theta_{i}(\beta)-\alpha\right) y^{2}+\left(\theta_{i}(\gamma)-\beta \alpha\right) y-\gamma \alpha,
\end{aligned}
$$

which is equivalent that $\alpha, \beta, \gamma$ satisfy $\theta_{i}(\beta)=\alpha, \theta_{i}(\gamma)=\beta \alpha$ and $\gamma \alpha=1$. From these and by $\theta_{i}^{3}=\operatorname{id}_{K_{i}}$, we deduce that $\beta=\theta_{i}^{2}\left(\theta_{i}(\beta)\right)=\theta_{i}^{2}(\alpha)$ and $\gamma=\theta_{i}^{2}\left(\theta_{i}(\gamma)\right)=\theta_{i}^{2}(\beta \alpha)=\theta_{i}(\alpha) \theta_{i}^{2}(\alpha)$, which implies $\alpha \theta_{i}(\alpha) \theta_{i}^{2}(\alpha)=1$. Then by the latter equation and (ii), we conclude that $\alpha \in \mathcal{G}_{i}$, and hence

$$
\gamma=\alpha^{-1} \text { and } \beta=\theta_{i}^{2}(\alpha) \text {. }
$$

Therefore, both the number of right divisors of $y^{3}-1$ in $K_{i}\left[y ; \theta_{i}\right]$ with degree 1 and the number of right divisors of $y^{3}-1$ in $K_{i}\left[y ; \theta_{i}\right]$ with degree 2 are equal to $1+q^{\frac{d_{i}}{3}}+q^{\frac{2 d_{i}}{3}}$.

(v) It follow from a direct calculation.

Now, by Lemma 3.2 and basic theory of skew cyclic codes we list all distinct left ideals of $\mathcal{R}_{i}$, i.e., skew $\theta_{i}$-cyclic codes over $K_{i}$ of length 3 , by the following theorem.

Theorem 3.3: Let $s+1 \leq i \leq s+t$. Then all distinct left ideals of $\mathcal{R}_{i}=K_{i}\left[y ; \theta_{i}\right] /\left\langle y^{3}-1\right\rangle$ are given by one of the following three cases:

(i) $C_{i, 0}=\{0\} ; C_{i, 3}=\mathcal{R}_{i}$ with $\left|C_{i, 3}\right|=q^{3 d_{i}}$, wt ${ }_{H}^{(i)}\left(C_{i, 3}\right)=1$ and $G_{C_{i, 3}}=I_{3}$ is a generator matrix of $C_{i, 3}$, where $I_{3}$ is the identity matrix of size $3 \times 3$.

(ii) $C_{i, 2, \alpha}=\mathcal{R}_{i}(-\alpha(x)+y)$, where $\alpha=\alpha(x) \in \mathcal{G}_{i}$.

Precisely, we have $\operatorname{dim}_{K_{i}}\left(C_{i, 2, \alpha}\right)=2,\left|C_{i, 2, \alpha}\right|=q^{2 d_{i}}$ and a generator matrix of $C_{i, 2, \alpha}$ is given by

$$
G_{C_{i, 2, \alpha}}=\left(\begin{array}{ccc}
-\alpha(x) & 1 & 0 \\
0 & -\theta_{i}(\alpha(x)) & 1
\end{array}\right) .
$$

Therefore, $C_{i, 2, \alpha}=\left\{(a, b) G_{C_{i, 2, \alpha}} \mid a, b \in K_{i}\right\}$ and $\mathrm{wt}_{H}^{(i)}\left(C_{i, 2, \alpha}\right)=2$. Hence $C_{i, 2, \alpha}$ is a MDS linear $[3,2,2]_{q^{d_{i}}}$-code over $K_{i}$.

(iii) $C_{i, 1, \alpha}=\mathcal{R}_{i}\left(\alpha(x)^{-1}+\theta_{i}^{2}(\alpha(x)) y+y^{2}\right)$, where $\alpha=\alpha(x) \in \mathcal{G}_{i}$.

Precisely, we have $\operatorname{dim}_{K_{i}}\left(C_{i, 1, \alpha}\right)=1,\left|C_{i, 1, \alpha}\right|=q^{d_{i}}$ and a generator matrix of $C_{i, 1, \alpha}$ is given by

$$
G_{C_{i, 1, \alpha}}=\left(\alpha(x)^{-1}, \theta_{i}^{2}(\alpha(x)), 1\right) .
$$

Hence $C_{i, 1, \alpha}=\left\{a G_{C_{i, 1, \alpha}} \mid a \in K_{i}\right\}$ and $\mathrm{wt}_{H}^{(i)}\left(C_{i, 1, \alpha}\right)=3$. Then $C_{i, 1, \alpha}$ is a MDS linear $[3,1,3]_{q^{d_{i}}}$-code over $K_{i}$.

Therefore, the number of left ideals of $\mathcal{R}_{i}$ is equal to $4+2 q^{\frac{d_{i}}{3}}+2 q^{\frac{2 d_{i}}{3}}$.

In the rest of this paper, for any $\alpha, \beta \in \mathcal{G}_{i}$, we denote

- $C_{i, 2, \alpha}=\mathcal{R}_{i}(-\alpha+y), C_{i, 1, \beta}=\mathcal{R}_{i}\left(\beta^{-1}+\theta_{i}^{2}(\beta) y+y^{2}\right)$

which are skew $\theta_{i}$-cyclic codes over $K_{i}$ of length 3. By Theorems 2.4 3.1 and 3.3, we deduce the following corollary.

Corollary 3.4: Using the notations in Section 2, denote

$$
\delta=\left|\left\{d_{i} \mid q^{d_{i}} \equiv 1(\bmod 3), 0 \leq i \leq s\right\}\right| .
$$

Then the number $N_{(m, 3, r ; q)}$ of left $G_{(m, 3, r)}$-codes over $\mathbb{F}_{q}$ is given by one of the following two cases:

(i) When $q \equiv 0(\bmod 3)$,

$$
N_{(m, 3, r ; q)}=4^{s+1} \prod_{i=s+1}^{s+t}\left(4+2 q^{\frac{d_{i}}{3}}+2 q^{\frac{2 d_{i}}{3}}\right) .
$$

(ii) When $q \not \equiv 0(\bmod 3)$,

$$
N_{(m, 3, r ; q)}=2^{\delta} 4^{s+1} \prod_{i=s+1}^{s+t}\left(4+2 q^{\frac{d_{i}}{3}}+2 q^{\frac{2 d_{i}}{3}}\right) .
$$


As the end of this section, we investigate the relationship between two left ideals of the ring $\mathcal{R}_{i}$ for $i=$ $s+1, \ldots, s+t$. To do this, we consider the relationship between two nontrivial monic right divisors of $y^{3}-1$ in $K_{i}\left[y, \theta_{i}\right]$ with different degrees.

Lemma 3.5: For any $\gamma \in \mathcal{G}_{i}$, denote

$$
\begin{gathered}
\phi_{i, \gamma}(X)=X^{q^{\frac{d_{i}}{3}}+1}+\gamma^{q^{\frac{2 d_{i}}{3}}} X+\gamma^{-1}, \\
\psi_{i, \gamma}(X)=\gamma^{q^{\frac{d_{i}}{3}}} X^{q^{\frac{d_{i}}{3}}+1}+\gamma^{-1} X^{q^{\frac{d_{i}}{3}}}+1 .
\end{gathered}
$$

Then both $\phi_{i, \gamma}(X)$ and $\psi_{i, \gamma}(X)$ have exactly $q^{\frac{d_{i}}{3}}+1$ roots and these roots are contained in $\mathcal{G}_{i}$.

Proof: As $\operatorname{gcd}\left(q, q^{\frac{d_{i}}{3}}+1\right)=1, \phi_{i, \gamma}(X)$ and $\psi_{i, \gamma}(X)$ have exactly $q^{\frac{d_{i}}{3}}+1$ roots in some extended field of $K_{i}$. We only need to prove that all these roots are contained in $\mathcal{G}_{i}$.

By $\gamma \in \mathcal{G}_{i} \subseteq K_{i}^{\times}$, we have $\gamma^{1+q^{\frac{d_{i}}{3}}+q^{\frac{2 d_{i}}{3}}}=1$ and $\gamma^{q^{d_{i}}}=\gamma$, which implies $\gamma^{q^{\frac{2 d_{i}}{3}}+1}=\gamma^{q^{-\frac{d_{i}}{3}}}$. Then from

$$
X^{q^{\frac{d_{i}}{3}}+1} \equiv-\left(\gamma^{q^{\frac{2 d_{i}}{3}}} X+\gamma^{-1}\right)\left(\bmod \phi_{i, \gamma}(X)\right)
$$

we deduce that

$$
\begin{aligned}
X^{q^{\frac{2 d_{i}}{3}}+q^{\frac{d_{i}}{3}}+1} & \equiv\left(X^{q^{\frac{d_{i}}{3}}+1}\right)^{q^{\frac{d_{i}}{3}}} X \\
& \equiv-\left(\gamma^{q^{\frac{2 d_{i}}{3}}} X+\gamma^{-1}\right)^{q^{\frac{d_{i}}{3}}} X \\
& \equiv-\left(\gamma^{q^{d_{i}}} X^{q^{\frac{d_{i}}{3}}+1}+\gamma^{q^{-\frac{d_{i}}{3}}} X\right) \\
& \equiv-\gamma\left(X^{q^{\frac{d_{i}}{3}}+1}+\gamma^{q^{\frac{2 d_{i}}{3}}} X\right) \\
& \equiv-\gamma \gamma^{-1}=1\left(\bmod \phi_{i, \gamma}(X)\right),
\end{aligned}
$$

which implies that $\phi_{i, \gamma}(X)$ is a factor of $X^{q^{\frac{2 d_{i}}{3}}+q^{\frac{d_{i}}{3}}+1}-1$. Hence $\alpha^{q^{\frac{2 d_{i}}{3}}+q^{\frac{d_{i}}{3}}+1}=1$ for any root $\alpha$ of $\phi_{i, \gamma}(X)$. Therefore, all roots of $\phi_{i, \gamma}(X)$ are contained in $\mathcal{G}_{i}$.

The reciprocal polynomial of $\psi_{i, \gamma}(X)$ is equal to

$$
\psi_{i, \gamma}^{*}(X)=X^{q^{\frac{d_{i}}{3}}+1}+\gamma^{-1} X+\gamma^{q^{\frac{d_{i}}{3}}} .
$$

Then by $X^{q^{\frac{d_{i}}{3}}+1} \equiv-\left(\gamma^{-1} X+\gamma^{q^{\frac{d_{i}}{3}}}\right)\left(\bmod \psi_{i, \gamma}^{*}(X)\right)$ and $\gamma^{-q^{\frac{d_{i}}{3}}-1}=\gamma^{q^{\frac{2 d_{i}}{3}}}$, it follows that

$$
\begin{aligned}
X^{q^{\frac{2 d_{i}}{3}}+q^{\frac{d_{i}}{3}}+1} & \equiv\left(X^{q^{\frac{d_{i}}{3}}+1}\right)^{q^{\frac{d_{i}}{3}}} X \\
& \equiv-\left(\gamma^{-1} X+\gamma^{q^{\frac{d_{i}}{3}}}\right)^{q^{\frac{d_{i}}{3}}} X \\
& \equiv-\left(\gamma^{-q^{\frac{d_{i}}{3}}} X^{q^{\frac{d_{i}}{3}}+1}+\gamma^{q^{\frac{2 d_{i}}{3}}} X\right) \\
& \equiv-\left(-\gamma^{-q^{\frac{d_{i}}{3}}}\left(\gamma^{-1} X+\gamma^{q^{\frac{d_{i}}{3}}}\right)+\gamma^{q^{\frac{2 d_{i}}{3}}} X\right) \\
& \equiv 1\left(\bmod \psi_{i, \gamma}^{*}(X)\right)
\end{aligned}
$$

which implies that $\psi_{i, \gamma}^{*}(x)$ is a factor of $X^{q^{\frac{2 d_{i}}{3}}+q^{\frac{d_{i}}{3}}+1}-1$. Since $X^{q^{\frac{2 d_{i}}{3}}+q^{\frac{d_{i}}{3}}+1}-1$ is self-reciprocal, we conclude that $\psi_{i, \gamma}(X)$ is also a factor of $X^{q^{\frac{2 d_{i}}{3}}+q^{\frac{d_{i}}{3}}+1}-1$. Therefore, all roots of $\psi_{i, \gamma}(X)$ are contained in $\mathcal{G}_{i}$.

Theorem 3.6: (i) Let $\alpha, \beta \in \mathcal{G}_{i}$. Then $C_{i, 1, \beta} \subset C_{i, 2, \alpha}$ if and only of $\alpha$ and $\beta$ satisfying the following equation

$$
\alpha \theta_{i}(\alpha) \beta+\alpha \beta \theta_{i}^{2}(\beta)+1=0 .
$$

(ii) For any $\beta \in \mathcal{G}_{i}$, there are $q^{\frac{d_{i}}{3}}+1$ codes $C_{i, 2, \alpha}$ containing $C_{i, 1, \beta}$ where $\alpha$ is given by one of the following two case:

$\alpha$ is a root of the polynomial $\phi_{i, \beta}(X)$ if $\theta_{i}(a(x))=a(x)^{q^{\frac{d_{i}}{3}}}$ for all $a(x) \in K_{i}$;

$\alpha$ is a root of the polynomial $\psi_{i, \beta}(X)$ if $\theta_{i}(a(x))=a(x)^{q^{\frac{2 d_{i}}{3}}}$ for all $a(x) \in K_{i}$. 
(iii) For any $\alpha \in \mathcal{G}_{i}$, there are $q^{\frac{d_{i}}{3}}+1$ codes $C_{i, 1, \beta}$ contained in $C_{i, 2, \alpha}$ where $\beta$ is given by one of the following two case:

$\beta$ is a root of the polynomial $\psi_{i, \alpha}(X)$ if $\theta_{i}(a(x))=a(x)^{q^{\frac{d_{i}}{3}}}$ for all $a(x) \in K_{i}$;

$\beta$ is a root of the polynomial $\phi_{i, \alpha}(X)$ if $\theta_{i}(a(x))=a(x)^{q^{\frac{2 d_{i}}{3}}}$ for all $a(x) \in K_{i}$.

Proof: (i) It is clear that $C_{i, 1, \beta} \subset C_{i, 2, \alpha}$ if and only if $-\alpha+y$ is a right divisor of $\beta^{-1}+\theta_{i}^{2}(\beta) y+y^{2}$.

Dividing $\beta^{-1}+\theta_{i}^{2}(\beta) y+y^{2}$ by $y-\alpha$ from right hand in the skew polynomial ring $K_{i}\left[y ; \theta_{i}\right]$, we have

$$
\begin{aligned}
& \beta^{-1}+\theta_{i}^{2}(\beta) y+y^{2} \\
= & \left(\theta_{i}(\alpha)+\theta_{i}^{2}(\beta)\right)(-\alpha+y)+\beta^{-1}+\alpha \theta_{i}(\alpha)+\alpha \theta_{i}^{2}(\beta) .
\end{aligned}
$$

Hence $-\alpha+y$ is a right divisor of $\beta^{-1}+\theta_{i}^{2}(\beta) y+y^{2}$ if and only if $\beta^{-1}+\alpha \theta_{i}(\alpha)+\alpha \theta_{i}^{2}(\beta)=0$, which is equivalent to that $\alpha$ and $\beta$ satisfy (5).

(ii) Let $\alpha \in \mathcal{G}_{i}$. We have one of the following two cases:

When $\theta_{i}(a(x))=a(x)^{q^{\frac{d_{i}}{3}}}$ for all $a(x) \in K_{i}$, it is clear that

$$
\alpha \theta_{i}(\alpha) \beta+\alpha \beta \theta_{i}^{2}(\beta)+1=\beta \phi_{i, \beta}(\alpha) .
$$

From this and by (i), we deduce that $C_{i, 1, \beta} \subset C_{i, 2, \alpha}$ if and only if $\phi_{i, \beta}(\alpha)=0$. Furthermore, by Lemma 3.5 we know that the $\phi_{i, \beta}(x)$ has exactly $q^{\frac{d_{i}}{3}}+1$ roots in $\mathcal{G}_{i}$.

When $\theta_{i}(a(x))=a(x)^{q^{\frac{2 d_{i}}{3}}}$ for all $a(x) \in K_{i}$, by $\alpha \theta_{i}(\alpha)=\alpha^{1+q^{\frac{2 d_{i}}{3}}}=q^{-q^{\frac{d_{i}}{3}}}$ we have

$$
\alpha \theta_{i}(\alpha) \beta+\alpha \beta \theta_{i}^{2}(\beta)+1=\alpha^{1+q^{\frac{2 d_{i}}{3}}} \beta \psi_{i, \beta}(\alpha) .
$$

From this and by (i), we deduce that $C_{i, 1, \beta} \subset C_{i, 2, \alpha}$ if and only if $\psi_{i, \beta}(\alpha)=0$. Furthermore, by Lemma 3.5 we know that the polynomial $\psi_{i, \beta}(x)$ has exactly $q^{\frac{d_{i}}{3}}+1$ roots in $\mathcal{G}_{i}$.

(iii) By (5) and Lemma 3.5, it can be proved similarly as that of (ii). Here, we omit the proof.

\section{The DuAl CODE OF ANY LeFT $G_{(m, 3, r)}$-CODE}

In this section, we give the dual code of any left $G_{(m, 3, r)}$-code over $\mathbb{F}_{q}$ and determine all self-orthogonal left $G_{(m, 3, r)}$-codes.

As in [?], the Euclidian inner product in $\mathbb{F}_{q}\left[G_{(m, 3, r)}\right]$ is defined as follows. For $\xi=\sum_{i=0}^{m-1} \sum_{j=0}^{2} a_{i, j} x^{i} y^{j}$ and $\eta=\sum_{i=0}^{m-1} \sum_{j=0}^{2} b_{i, j} x^{i} y^{j}$ in $\mathbb{F}_{q}\left[G_{(m, 3, r)}\right]$, we set

$$
[\xi, \eta]_{E}=\sum_{i=0}^{m-1} \sum_{j=0}^{2} a_{i, j} b_{i, j} \in \mathbb{F}_{q} .
$$

The Euclidian dual code of a left $G_{(m, 3, r)}$-code $\mathcal{C}$ over $\mathbb{F}_{q}$ is defined by

$$
\mathcal{C}^{\perp_{E}}=\left\{\xi \in \mathbb{F}_{q}\left[G_{(m, 3, r)}\right] \mid[\xi, \eta]_{E}=0, \forall \eta \in \mathcal{C}\right\} .
$$

$\mathcal{C}$ is said to be self-orthogonal if $\mathcal{C} \subseteq \mathcal{C}^{\perp_{E}}$.

For any $\xi(y)=\sum_{i=0}^{m-1} \sum_{j=0}^{2} a_{i, j} x^{i} y^{j} \in \mathbb{F}_{q}\left[G_{(m, 3, r)}\right]$, as in [?] we define the conjugation $\mu$ on $\mathbb{F}_{q}\left[G_{(m, 3, r)}\right]$ by

$$
\begin{aligned}
\mu(\xi(y)) & =\sum_{i=0}^{m-1} \sum_{j=0}^{2} a_{i, j} y^{-j} x^{-i} \\
& =a_{0}\left(x^{-1}\right)+y^{2} a_{1}\left(x^{-1}\right)+y a_{2}\left(x^{-1}\right),
\end{aligned}
$$

where $a_{j}(x)=\sum_{i=0}^{m-1} a_{i, j} x^{i}$ and $a_{j}\left(x^{-1}\right)=a_{0, j}+\sum_{i=1}^{m-1} a_{i, j} x^{m-i}$ for $j=0,1,2$. It can be verify easily that

$$
\mu(\xi+\eta)=\mu(\xi)+\mu(\eta) \text { and } \mu(\xi \eta)=\mu(\eta) \mu(\xi)
$$

for all $\xi, \eta \in \mathbb{F}_{q}\left[G_{(m, 3, r)}\right]$. Moreover, we have the following

Lemma 4.1: (i) The map $\mu$ defined by (6) is an $\mathbb{F}_{q^{-}}$-algebra anti-automorphism of $\mathbb{F}_{q}\left[G_{(m, 3, r)}\right]$ satisfying $\mu^{-1}=\mu$. 
(ii) For any $\xi, \eta \in \mathbb{F}_{q}\left[G_{(m, 3, r)}\right]$, we have $[\xi, \eta]_{E}=0$ if $\xi \cdot \mu(\eta)=0$ in the ring $\mathbb{F}_{q}\left[G_{(m, 3, r)}\right]$.

(iii) Let $\mathcal{C}$ be a left $G_{(m, 3, r)}$-code over $\mathbb{F}_{q}$ and $\mathcal{B}$ a right ideal of $\mathbb{F}_{q}\left[G_{(m, 3, r)}\right]$. Then

$$
\mu(\mathcal{B}) \subseteq \mathcal{C}^{\perp_{E}} \text { if } \mathcal{C} \cdot \mathcal{B}=\{0\} \text { in } \mathbb{F}_{q}\left[G_{(m, 3, r)}\right]
$$

Proof: (ii) For $\xi=\sum_{i=0}^{m-1} \sum_{j=0}^{2} a_{i, j} x^{i} y^{j}$ and $\eta=\sum_{i=0}^{m-1} \sum_{j=0}^{2} b_{i, j} x^{i} y^{j}$ in $\mathbb{F}_{q}\left[G_{(m, 3, r)}\right]$, by ord $(x)=m$ and $\operatorname{ord}(y)=3$ we deduce that

$$
\begin{aligned}
\xi \cdot \mu(\eta) & =\left(\sum_{i=0}^{m-1} \sum_{j=0}^{2} a_{i, j} x^{i} y^{j}\right)\left(\sum_{k=0}^{m-1} \sum_{l=0}^{2} b_{k, l} y^{3-l} x^{m-k}\right) \\
& =[\xi, \eta]_{E}+\sum_{0 \leq i \leq m-1,0 \leq j \leq 2, i+j \neq 0} c_{i, j} x^{i} y^{j}
\end{aligned}
$$

for some $c_{i, j} \in \mathbb{F}_{q}$. Hence $[\xi, \eta]_{E}=0$ if $\xi \cdot \mu(\eta)=0$ in the ring $\mathbb{F}_{q}\left[G_{(m, 3, r)}\right]$.

(iii) For any $\beta \in \mathcal{B}$ and $\eta \in \mathcal{C}$, by $\mathcal{C} \cdot \mathcal{B}=\{0\}$ we have $\eta \beta=0$, which implies $\mu(\beta) \cdot \mu(\eta)=0$ by (i), and so $[\mu(\beta), \eta]_{E}=0$ by (ii). From this we deduce that $\mu(\beta) \in \mathcal{C}^{\perp_{E}}$. Therefore, $\mu(\mathcal{B}) \subseteq \mathcal{C}^{\perp_{E}}$.

By the identification of $\mathbb{F}_{q}\left[G_{(m, 3, r)}\right]$ with $\mathcal{A}[y ; \theta] /\left\langle y^{3}-1\right\rangle$, we see that $\mathcal{A}=\mathbb{F}_{q}[x] /\left\langle x^{m}-1\right\rangle$ is a subring of $\mathbb{F}_{q}\left[G_{(m, 3, r)}\right]$. In the following, we consider the restriction of $\mu$ on $\mathcal{A}$. In order to simplify the notation, we still denote this restriction by $\mu$. Obviously, we have

$$
\mu(a(x))=a\left(x^{-1}\right)=\sum_{i=0}^{m-1} a_{i} x^{-i}=a_{0}+\sum_{i=1}^{m-1} a_{i} x^{m-i}
$$

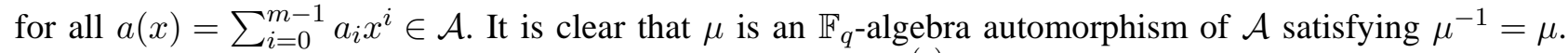

Using the notations of Section 2, we know that $J(i)=J_{k_{i}}^{(q)}, 0 \leq i \leq s+t$, are the all distinct $q$-cyclotomic cosets modulo $m$. By Lemma 2.2, we have one of the following two cases:

$\diamond 0 \leq i \leq s$. In this case, we have $\theta(j)=r j \equiv j(\bmod m)$ for all $j \in J(i)=J_{k_{i}}^{(q)}$. Then it is clear that $-J(i)=\{-j \mid j \in J(i)\}(\bmod m)$ is a $q$-cyclotomic coset modulo $m$ satisfying $\theta(-j)=-r j \equiv-j(\bmod m)$ for all $j \in J(i)$. Hence $-J(i)$ is also a $q$-cyclotomic coset modulo $m$ satisfying Condition (I) in Lemma 2.2, Therefore, there is a unique integer $i^{\prime}, 0 \leq i^{\prime} \leq s$, such that $-J(i)=J\left(i^{\prime}\right)$.

$\diamond s+1 \leq i \leq s+t$. In this case, we have $\theta(j) \in J(i)$ and $\theta(j) \not \equiv j(\bmod m)$ for all $j \in J(i)=J_{k_{i}}^{(q)}$. Then it is clear that $-J(i)$ is a $q$-cyclotomic coset modulo $m$ satisfying $\theta(-j)=-\theta(j) \in-J(i)$ and $\theta(-j) \not \equiv-j(\bmod$ $m$ ) for all $j \in J(i)$. Hence $-J(i)$ is also a $q$-cyclotomic coset modulo $m$ satisfying Condition (II) in Lemma 2.2. Therefore, there is a unique integer $i^{\prime}, s+1 \leq i^{\prime} \leq s+t$, such that $-J(i)=J\left(i^{\prime}\right)$.

We also use $\mu$ to denote this map $i \mapsto i^{\prime}$, i.e., $\mu(i)=i^{\prime}$. Whether $\mu$ denotes the automorphism of $\mathcal{A}$ or this map on the set $\{0,1, \ldots, s+t\}$ is determined by context. The next lemma shows the compatibility of the two uses of $\mu$.

Lemma 4.2: Using the notations above, the following assertions hold.

(i) $\mu$ is a permutation on $\{0,1, \ldots, s+t\}$ satisfying $\mu^{-1}=\mu, \mu(0)=0,1 \leq \mu(i) \leq s$ for all $1 \leq i \leq s$ and $s+1 \leq \mu(i) \leq s+t$ for all $s+1 \leq i \leq s+t$.

(ii) After a rearrangement of $J(0), J(1) \ldots, J(s+t)$, there are nonnegative integers $s_{1}, s_{2}, t_{1}, t_{2}$ satisfying the following conditions:

- $s=s_{1}+2 s_{2}, \mu(i)=i$ for all $1 \leq i \leq s_{1}, \mu(i)=i+s_{2}$ and $\mu\left(i+s_{2}\right)=i$ for all $s_{1}+1 \leq i \leq s_{1}+s_{2}$;

- $t=t_{1}+2 t_{2}, \mu(i)=i$ for all $s+1 \leq i \leq s+t_{1}, \mu(i)=i+t_{2}$ and $\mu\left(i+t_{2}\right)=i$ for all $s+t_{1}+1 \leq i \leq s+t_{1}+t_{2}$.

(iii) $\mu\left(\varepsilon_{i}(x)\right)=\varepsilon_{\mu(i)}(x)$ and $\mu\left(\mathcal{A}_{i}\right)=\mathcal{A}_{\mu(i)}$ for all $i=0,1, \ldots, s+t$.

(iv) Let $\mu$ be the map defined by $\mu(a(x))=a\left(x^{-1}\right)=a\left(x^{m-1}\right)\left(\bmod f_{\mu(i)}(x)\right)$ for all $a(x) \in K_{i}=$ $\mathbb{F}_{q}[x] /\left\langle f_{i}(x)\right\rangle$. Then $\mu$ is an $\mathbb{F}_{q}$-algebra isomorphism from $K_{i}$ onto $K_{\mu(i)}=\mathbb{F}_{q}[x] /\left\langle f_{\mu(i)}(x)\right\rangle$ satisfying $\mu \theta_{i}=\theta_{\mu(i)} \mu$.

(v) Let $0 \leq i \leq s+t$. Using the notations of Theorem 2.4 (ii), the $\mathbb{F}_{q}$-algebra anti-automorphism $\mu$ of $\mathbb{F}_{q}\left[G_{(m, 3, r)}\right]$ induces an $\mathbb{F}_{q}$-algebra anti-isomorphism $\varphi_{\mu(i)}^{-1} \mu \varphi_{i}$ from $\mathcal{R}_{i}$ onto $\mathcal{R}_{\mu(i)}$. We denote this anti-isomorphism by $\mu$ as well. Then for any $\alpha(y)=a(x)+b(x) y+c(x) y^{2} \in \mathcal{R}_{i}$ where $a(x), b(x), c(x) \in K_{i}$, we have

$$
\mu(\alpha(y))=a\left(x^{-1}\right)+y^{2} b\left(x^{-1}\right)+y c\left(x^{-1}\right) .
$$


Proof: (i) follows from the definition of the map $\mu$, and (ii) follows from (i).

(iii) It is clear that $\mu\left(\varepsilon_{i}(x)\right)=\frac{1}{m} \sum_{l=0}^{m-1}\left(\sum_{j \in J(i)} \zeta^{-j l}\right) x^{-l}$ by (3) in Section 2. From this, by $\mathbb{Z}_{m}=-\mathbb{Z}_{m}$ and $J(\mu(i))=-J(i)=\{-j \mid j \in J(i)\}$ we deduce that

$$
\begin{aligned}
\mu\left(\varepsilon_{i}(x)\right) & =\frac{1}{m} \sum_{l=0}^{m-1}\left(\sum_{j \in J(i)} \zeta^{-(-j)(-l)}\right) x^{-l} \\
& =\frac{1}{m} \sum_{k=0}^{m-1}\left(\sum_{j^{\prime} \in J(\mu(i))} \zeta^{-j^{\prime} k}\right) x^{k} \\
& =\varepsilon_{\mu(i)}(x) .
\end{aligned}
$$

Hence $\mu\left(\mathcal{A}_{i}\right)=\mu\left(\mathcal{A} \varepsilon_{i}(x)\right)=\mu(\mathcal{A}) \mu\left(\varepsilon_{i}(x)\right)=\mathcal{A} \varepsilon_{\mu(i)}(x)=\mathcal{A}_{\mu(i)}$ by Lemma 2.3 (ii).

(iv) By (iii), we know that $\mu$ induces an $\mathbb{F}_{q}$-algebra isomorphism from $\mathcal{A}_{i}$ onto $\mathcal{A}_{\mu(i)}$. Then by Lemma 2.3 (iii), we see that $\varphi_{\mu(i)}^{-1} \mu \varphi_{i}$ is an $\mathbb{F}_{q}$-algebra isomorphism from $K_{i}$ onto $K_{\mu(i)}$. For any $a(x) \in K_{i}$, by Equation (3) in Section 2 we have $\varepsilon_{\mu(i)}(x) \equiv 1\left(\bmod f_{\mu(i)}(x)\right)$, which implies

$$
\begin{aligned}
\left(\varphi_{\mu(i)}^{-1} \mu \varphi_{i}\right)(a(x)) & =\varphi_{\mu(i)}^{-1} \mu\left(\varepsilon_{i}(x) a(x)\right) \\
& =\varphi_{\mu(i)}^{-1}\left(\varepsilon_{\mu(i)}(x) a\left(x^{-1}\right)\right) \\
& =a\left(x^{-1}\right)\left(\bmod f_{\mu(i)}(x)\right) .
\end{aligned}
$$

Since we denote $\varphi_{\mu(i)}^{-1} \mu \varphi_{i}$ by $\mu$ as well, the map $\mu: a(x) \mapsto a\left(x^{-1}\right)\left(\bmod f_{\mu(i)}(x)\right)$ is an $\mathbb{F}_{q^{-a l g e b r a}}$ isomorphism from $K_{i}$ onto $K_{\mu(i)}$. Moreover, for any $a(x) \in K_{i}$ by Lemma 2.3 (v) and $a\left(x^{-1}\right) \in K_{\mu(i)}$ it follows that

$$
\begin{aligned}
\left(\mu \theta_{i}\right)(a(x)) & =\mu\left(a\left(x^{r}\right)\right)=a\left(x^{-r}\right)=\theta_{\mu(i)}\left(a\left(x^{-1}\right)\right) \\
& =\left(\theta_{\mu(i)} \mu\right)(a(x)) .
\end{aligned}
$$

Hence $\mu \theta_{i}=\theta_{\mu(i)} \mu$.

(v) By (iii) and Theorem 2.4(ii), we have the following commutative diagram form ring isomorphisms:

$$
\begin{array}{rcc}
\mathcal{R}_{i} & \stackrel{\varphi_{i}}{\longrightarrow} & \mathcal{A}_{i}\left[y ;\left.\theta\right|_{\mathcal{A}_{i}}\right] /\left\langle\varepsilon_{i}(x)\left(y^{3}-1\right)\right\rangle \\
\varphi_{\mu(i)}^{-1} \mu \varphi_{i} \downarrow & & \downarrow \mu \\
\mathcal{R}_{\mu(i)} & \stackrel{\varphi_{\mu(i)}}{\longrightarrow} & \mathcal{A}_{\mu(i)}\left[y ;\left.\theta\right|_{\mathcal{A}_{\mu(i)}}\right] /\left\langle\varepsilon_{\mu(i)}(x)\left(y^{3}-1\right)\right\rangle
\end{array}
$$

As we write $\varphi_{\mu(i)}^{-1} \mu \varphi_{i}$ by $\mu$, for any $a(x), b(x), c(x) \in K_{i}$ by the identification of $\mathbb{F}_{q}\left[G_{(m, 3, r)}\right]$ with $\mathcal{A}[y ; \theta] /\left\langle y^{3}-1\right\rangle$, $\varepsilon_{i}\left(x^{-1}\right)=\mu\left(\varepsilon_{i}(x)\right)=\varepsilon_{\mu(i)}(x)$, Equation (6) and $y \varepsilon_{\mu(i)}(x)=\theta\left(\varepsilon_{\mu(i)}(x)\right) y=\varepsilon_{\mu(i)}(x) y$, we deduce that

$$
\begin{aligned}
& \mu\left(a(x)+b(x) y+c(x) y^{2}\right) \\
= & \left(\varphi_{\mu(i)}^{-1} \mu\right)\left(\varphi_{i}\left(a(x)+b(x) y+c(x) y^{2}\right)\right) \\
= & \varphi_{\mu(i)}^{-1}\left(\mu\left(\varepsilon_{i}(x) a(x)+\varepsilon_{i}(x) b(x) y+\varepsilon_{i}(x) c(x) y^{2}\right)\right) \\
= & \varphi_{\mu(i)}^{-1}\left(\varepsilon_{i}\left(x^{-1}\right) a\left(x^{-1}\right)+y^{2} \varepsilon_{i}\left(x^{-1}\right) b\left(x^{-1}\right)\right. \\
& \left.+y \varepsilon_{i}\left(x^{-1}\right) c\left(x^{-1}\right)\right) \\
= & \varphi_{\mu(i)}^{-1}\left(\varepsilon_{\mu(i)}(x)\left(a\left(x^{-1}\right)+y^{2} b\left(x^{-1}\right)+y c\left(x^{-1}\right)\right)\right) \\
= & a\left(x^{-1}\right)+y^{2} b\left(x^{-1}\right)+y c\left(x^{-1}\right)
\end{aligned}
$$

by (iv).

Corollary 4.3: For any $\alpha(x) \in \mathcal{G}_{i}$, we denote

$$
\widehat{\alpha}(x)=\left(\alpha\left(x^{m-1}\right)\right)^{q^{\frac{2 d_{i}}{3}}+q^{\frac{d_{i}}{3}}}\left(\bmod f_{\mu(i)}(x)\right) .
$$

Using the notations of Lemma 4.2(iv), we have that $\widehat{\alpha}(x)=\left(\alpha\left(x^{-1}\right)\right)^{-1}=(\mu(\alpha(x)))^{-1} \in \mathcal{G}_{\mu(i)}, \alpha\left(x^{-1}\right) \widehat{\alpha}(x)=1$ and $\alpha(x)=\left(\widehat{\alpha}\left(x^{-1}\right)\right)^{-1}$. 
Proof: As $\alpha(x) \in \mathcal{G}_{i}$, we see that $\alpha(x)$ is an element of $K_{i}=\mathbb{F}_{q}[x] /\left\langle f_{i}(x)\right\rangle$ satisfying $(\alpha(x))^{q^{\frac{2 d_{i}}{3}}+q^{\frac{d_{i}}{3}}+1}=1$. By Lemma 4.2 (iv), we know that $\mu$ is an $\mathbb{F}_{q}$-algebra isomorphism from $K_{i}$ onto $K_{\mu(i)}=\mathbb{F}_{q}[x] /\left\langle f_{\mu(i)}(x)\right\rangle$. Hence $(\mu(\alpha(x)))^{-1} \in K_{\mu(i)}$ and $(\mu(\alpha(x)))^{q^{\frac{2 d_{i}}{3}}+q^{\frac{d_{i}}{3}}+1}=1$ in $K_{\mu(i)}$, which implies $\mu(\alpha(x)) \in \mathcal{G}_{\mu(i)}$, and so $\left(\mu(\alpha(x))^{-1} \in\right.$ $\mathcal{G}_{\mu(i)}$. Finally, by $f_{\mu(i)}(x) \mid\left(x^{m}-1\right)$ it follows that

$$
\begin{aligned}
\widehat{\alpha}(x) & =\left(\alpha\left(x^{-1}\right)\right)^{q^{\frac{2 d_{i}}{3}}+q^{\frac{d_{i}}{3}}}=(\mu(\alpha(x)))^{q^{\frac{2 d_{i}}{3}}+q^{\frac{d_{i}}{3}}} \\
& =(\mu(\alpha(x)))^{-1}
\end{aligned}
$$

in $K_{\mu(i)}$. Then $\alpha(x)=(\mu(\widehat{\alpha}(x)))^{-1}=\left(\widehat{\alpha}\left(x^{-1}\right)\right)^{-1}$.

For any integer $i, 0 \leq i \leq s+t$, and $g(y), h(y) \in \mathcal{R}_{i}=K_{i}\left[y ; \theta_{i}\right] /\left\langle y^{3}-1\right\rangle$, in the following we define

$$
g(y) \sim_{l} h(y) \text { if } g(y)=\alpha h(y) \text { for some } \alpha \in \mathcal{R}_{i}^{\times},
$$

where $\mathcal{R}_{i}^{\times}$is the set of invertible elements in $\mathcal{R}_{i}$. It is clear that $\mathcal{R}_{i} g(y)=\mathcal{R}_{i} h(y)$ if $g(y)=\alpha h(y)$.

Lemma 4.4: For any integer $i, 0 \leq i \leq s+t$, we have the following conclusions:

(i) $\mu\left(y^{2}+y+1\right) \sim_{l} y^{2}+y+1, \mu\left(y^{3}-1\right) \sim_{l} y^{3}-1$ and $\mu\left((y-1)^{j}\right) \sim_{l}(y-1)^{j}$ for all $j=0,1,2$.

(ii) Let $0 \leq i \leq s$ and $q^{d_{i}} \equiv 1(\bmod 3)$. Then $\mu\left(y-\omega_{i}(x)\right) \sim_{l} y-\omega_{i}\left(x^{-1}\right)^{2}$ and $\mu\left(y-\omega_{i}(x)^{2}\right) \sim_{l} y-\omega_{i}\left(x^{-1}\right)$ in $\mathcal{R}_{\mu(i)}$.

(iii) Let $s+1 \leq i \leq s+t$ and $\alpha(x) \in \mathcal{G}_{i}$. Then

$$
\begin{gathered}
\mu(-\alpha(x)+y) \sim_{l}-\theta_{\mu(i)}(\widehat{\alpha}(x))+y \\
\mu\left(\alpha(x)^{-1}+\theta_{i}^{2}(\alpha(x)) y+y^{2}\right) \\
\sim_{l} \quad\left(\theta_{\mu(i)}^{2}(\widehat{\alpha}(x))\right)^{-1}+\theta_{\mu(i)}^{2}\left(\theta_{\mu(i)}^{2}(\widehat{\alpha}(x))\right) y+y^{2} .
\end{gathered}
$$

Proof: (i) By Equation (7) and $y^{3}=1$, it follows that $\mu(y-1)=y^{2}-1=\left(-y^{2}\right)(y-1)$ where $-y^{2} \in \mathcal{R}_{i}^{\times}$. The other conclusion can be verified similarly.

(ii) Since $\omega_{i}(x)^{3}=1$ and $\mu$ is a ring isomorphism from $\mathcal{R}_{i}$ onto $\mathcal{R}_{\mu(i)}$ by Lemma 4.2(v), it follows that $\omega_{i}\left(x^{-1}\right)^{3}=\left(\mu\left(\omega_{i}(x)\right)\right)^{3}=1$. As $-\omega_{i}\left(x^{-1}\right) y^{2} \in \mathcal{R}_{\mu(i)}^{\times}$, we have

$$
\begin{aligned}
\mu\left(y-\omega_{i}(x)\right) & =-\omega_{i}\left(x^{-1}\right)+y^{2} \\
& =\left(-\omega_{i}\left(x^{-1}\right) y^{2}\right)\left(y-\omega_{i}\left(x^{-1}\right)^{2}\right) \\
& \sim_{l} y-\omega_{i}\left(x^{-1}\right)^{2} .
\end{aligned}
$$

Similarly, one can verify that $\mu\left(y-\omega_{i}(x)^{2}\right) \sim_{l} y-\omega_{i}\left(x^{-1}\right)$.

(iii) By (7), Lemma 4.2(v) and Corollary 4.3 we have

$$
\begin{aligned}
\mu(-\alpha(x)+y) & =-\alpha\left(x^{-1}\right)+y^{2} \\
& =-\mu(\alpha(x))\left(1-\widehat{\alpha}(x) y^{2}\right) \\
& =-\mu(\alpha(x))\left(1-y^{2} \theta_{\mu(i)}(\widehat{\alpha}(x))\right) \\
& =-\mu(\alpha(x)) y^{2} \cdot y\left(1-y^{2} \theta_{\mu(i)}(\widehat{\alpha}(x))\right) \\
& =-\mu(\alpha(x)) y^{2}\left(-\theta_{\mu(i)}(\widehat{\alpha}(x))+y\right),
\end{aligned}
$$

where $-\mu(\alpha(x)) y^{2} \in \mathcal{R}_{\mu(i)}^{\times}$and $\theta_{\mu(i)}(\widehat{\alpha}(x)) \in \mathcal{G}_{\mu(i)}$ by Corollary 4.3 . Similarly, we have

$$
\begin{aligned}
& \mu\left(\alpha(x)^{-1}+\theta_{i}^{2}(\alpha(x)) y+y^{2}\right) \\
= & \alpha\left(x^{-1}\right)^{-1}+y^{2} \theta_{\mu(i)}^{2}\left(\alpha\left(x^{-1}\right)\right)+y \\
= & y^{2} \cdot y\left(\widehat{\alpha}(x)+y^{2} \theta_{\mu(i)}^{2}\left(\widehat{\alpha}(x)^{-1}\right)+y\right) \\
= & y^{2}\left(\theta_{\mu(i)}^{2}\left(\widehat{\alpha}(x)^{-1}\right)+\theta_{\mu(i)}(\widehat{\alpha}(x)) y+y^{2}\right)
\end{aligned}
$$


where $y^{2} \in \mathcal{R}_{\mu(i)}^{\times}, \theta_{\mu(i)}^{2}\left(\widehat{\alpha}(x)^{-1}\right)=\left(\theta_{\mu(i)}^{2}(\widehat{\alpha}(x))\right)^{-1} \in \mathcal{G}_{\mu(i)}$ by Corollary 4.3 and $\theta_{\mu(i)}(\widehat{\alpha}(x))=\theta_{\mu(i)}^{2}\left(\theta_{\mu(i)}^{2}(\widehat{\alpha}(x))\right)$.

Now, we give the dual code of any left $G_{(m, 3, r)}$-code over $\mathbb{F}_{q}$ by the following theorem.

Theorem 4.5: Let $\mathcal{C}=\oplus_{i=0}^{s+t}\left(\mathcal{A}_{i} \square_{\varphi_{i}} C_{i}\right)$ be a left $G_{(m, 3, r)}$-code over $\mathbb{F}_{q}$, where $C_{i}$ is a left ideal of the ring $\mathcal{R}_{i}=K_{i}\left[y ; \theta_{i}\right] /\left\langle y^{3}-1\right\rangle$ given by Theorems 3.1 and 3.3. Then the dual code $\mathcal{C}^{\perp_{E}}$ of $\mathcal{C}$ is also a left $G_{(m, 3, r)}$-code over $\mathbb{F}_{q}$. Precisely, we have

$$
\mathcal{C}^{\perp_{E}}=\bigoplus_{i=0}^{s+t}\left(\mathcal{A}_{i} \square_{\varphi_{i}} D_{i}\right),
$$

where $D_{i}$ is a left ideal of $\mathcal{R}_{i}$ given by one of the following cases:

(i) Let $0 \leq i \leq s$. Then $\mathcal{R}_{i}=K_{i}[y] /\left\langle y^{3}-1\right\rangle$ and $D_{i}$ is given by one of the following subcases:

(i-1) Let $q \equiv 0(\bmod 3)$. Then $D_{\mu(i)}=\mathcal{R}_{\mu(i)} \frac{(y-1)^{3}}{g(y)}$, if $C_{i}=\mathcal{R}_{i} g(y)$ where $g(y) \in\left\{1, y-1,(y-1)^{2},(y-1)^{3}\right\}$.

(i-2) Let $q^{d_{i}} \equiv 2(\bmod 3)$. Then $D_{\mu(i)}=\mathcal{R}_{\mu(i)} \frac{y^{3}-1}{g(y)}$, if $C_{i}=\mathcal{R}_{i} g(y)$ where $g(y) \in\left\{1, y-1, y^{2}+y+1, y^{3}-1\right\}$.

(i-3) Let $q^{d_{i}} \equiv 1(\bmod 3)$. Then $D_{\mu(i)}=\mathcal{R}_{\mu(i)} \vartheta(y)$ if $C_{i}=\mathcal{R}_{i} g(y)$, where the pair $(g(y), \vartheta(y))$ of polynomials is given by the following table:

\begin{tabular}{l|l}
\hline$g(y)$ & $\vartheta(y)\left(\bmod f_{\mu(i)}(x)\right)$ \\
\hline 1 & $y^{3}-1$, \\
$y-1$ & $y^{2}+y+1$ \\
$y-\omega_{i}(x)$ & $(y-1)\left(y-\omega_{i}\left(x^{-1}\right)\right)$ \\
$y-\omega_{i}(x)^{2}$ & $(y-1)\left(y-\omega_{i}\left(x^{-1}\right)^{2}\right)$ \\
$y^{2}+y+1$ & $y-1$ \\
$(y-1)\left(y-\omega_{i}(x)^{2}\right)$ & $y-\omega_{i}\left(x^{-1}\right)^{2}$ \\
$(y-1)\left(y-\omega_{i}(x)\right)$ & $y-\omega_{i}\left(x^{-1}\right)$ \\
$y^{3}-1$ & 1 \\
\hline
\end{tabular}

(ii) Let $s+1 \leq i \leq s+t$. Then $\mathcal{R}_{i}=K_{i}\left[y ; \theta_{i}\right] /\left\langle y^{3}-1\right\rangle$ and $D_{i}$ is given by one of the following subcases: (ii-1) $D_{\mu(i)}=\mathcal{R}_{\mu(i)}\left(-\theta_{\mu(i)}(\widehat{\alpha}(x))+y\right)=C_{\mu(i), 2, \theta_{\mu(i)}(\widehat{\alpha})}$ and a generator matrix of $D_{\mu(i)}$ is given by

$$
G_{D_{\mu(i)}}=\left(\begin{array}{ccc}
-\theta_{\mu(i)}(\widehat{\alpha}(x)) & 1 & 0 \\
0 & -\theta_{\mu(i)}\left(\theta_{\mu(i)}(\widehat{\alpha}(x))\right) & 1
\end{array}\right)
$$

as a linear code over $K_{\mu(i)}$ of length 3 , if $C_{i}=C_{i, 1, \alpha}=\mathcal{R}_{i}\left(\alpha(x)^{-1}+\theta_{i}^{2}(\alpha(x)) y+y^{2}\right)$ where $\alpha(x) \in \mathcal{G}_{i}$. by

(ii-2) $D_{\mu(i)}=\mathcal{R}_{\mu(i)}\left(\theta_{\mu(i)}^{2}\left(\widehat{\alpha}(x)^{-1}\right)+\theta_{\mu(i)}(\widehat{\alpha}(x)) y+y^{2}\right)=C_{\mu(i), 1, \theta_{\mu(i)}^{2}(\widehat{\alpha})}$ and a generator matrix of $D_{\mu(i)}$ is given

$$
G_{D_{\mu(i)}}=\left(\left(\theta_{\mu(i)}^{2}(\widehat{\alpha}(x))\right)^{-1}, \theta_{\mu(i)}^{2}\left(\theta_{\mu(i)}^{2}(\widehat{\alpha}(x))\right), 1\right)
$$

as a linear code over $K_{\mu(i)}$ of length 3 , if $C_{i}=C_{i, 2, \alpha}=\mathcal{R}_{i}(-\alpha(x)+y)$ where $\alpha(x) \in \mathcal{G}_{i}$.

(ii-3) $D_{\mu(i)}=\{0\}$ if $C_{i}=\mathcal{R}_{i} ; D_{\mu(i)}=\mathcal{R}_{\mu(i)}$ if $C_{i}=\{0\}$.

Proof: Let $K_{i}=\mathbb{F}_{q}[x] /\left\langle f_{i}(x)\right\rangle, \mathcal{R}_{i}=K_{i}\left[y ; \theta_{i}\right] /\left\langle y^{3}-1\right\rangle$ and $B_{i}$ be an right ideal of the ring $\mathcal{R}_{i}=$ $K_{i}\left[y ; \theta_{i}\right] /\left\langle y^{3}-1\right\rangle$ given by one of the following two cases:

(A) Let $0 \leq i \leq s$. Then $\theta_{i}=\mathrm{id}_{K_{i}}, \mathcal{R}_{i}=K_{i}[y] /\left\langle y^{3}-1\right\rangle$ and $B_{i}$ is given by one of the following three subcases.

(A-1) Let $q \equiv 0(\bmod 3)$. Then $B_{i}=\frac{y^{3}-1}{g(y)} \mathcal{R}_{i}$ if $C_{i}=\mathcal{R}_{i} g(y)$ where $g(y) \in\left\{1, y-1,(y-1)^{2}, y^{3}-1\right\}$.

(A-2) Let $q^{d_{i}} \equiv 2(\bmod 3)$. Then $B_{i}=\frac{y^{3}-1}{g(y)} \mathcal{R}_{i}$ if $C_{i}=\mathcal{R}_{i} g(y)$ where $g(y) \in\left\{1, y-1, y^{2}+y+1, y^{3}-1\right\}$.

(A-3) Let $q^{d_{i}} \equiv 1(\bmod 3)$. Then $B_{i}=\frac{y^{3}-1}{g(y)} \mathcal{R}_{i}$ if $C_{i}=\mathcal{R}_{i} g(y)$ where $g(y) \in\left\{1, y-1, y-\omega_{i}(x), y-\omega_{i}(x)^{2}, y^{2}+\right.$ $\left.y+1,(y-1)\left(y-\omega_{i}(x)^{2}\right),(y-1)\left(y-\omega_{i}(x)\right), y^{3}-1\right\}$.

(B) Let $s+1 \leq i \leq s+t$. Then $B_{i}$ is given by one of the following four subcases.

(B-1) $B_{i}=(-\alpha(x)+y) \mathcal{R}_{i}$, if $C_{i}=\mathcal{R}_{i}\left(\alpha(x)^{-1}+\theta_{i}^{2}(\alpha(x)) y+y^{2}\right)$ where $\alpha(x) \in \mathcal{G}_{i}$.

(B-2) $B_{i}=\left(\alpha(x)^{-1}+\theta_{i}^{2}(\alpha(x)) y+y^{2}\right) \mathcal{R}_{i}$, if $C_{i}=\mathcal{R}_{i}(-\alpha(x)+y)$ where $\alpha(x) \in \mathcal{G}_{i}$.

(B-3) $B_{i}=\{0\}$, if $C_{i}=\mathcal{R}_{i}$; $B_{i}=\mathcal{R}_{i}$, if $C_{i}=\{0\}$. 
By Theorems 3.1 and 3.3, Lemma 3.2(v) and direct calculations, one can easily verify that

$$
C_{i} \cdot B_{i}=\{0\} \text { in } \mathcal{R}_{i}, i=0,1, \ldots, s+t .
$$

For any integer $0 \leq i \leq s+t$, let $D_{\mu(i)}=\mu\left(B_{i}\right)$. By Lemma 4.2(v) we see that $D_{\mu(i)}$ is a left ideal of $\mathcal{R}_{\mu(i)}$. Let

$$
\mathcal{D}=\sum_{i=0}^{s+t} \mathcal{A}_{i} \square_{\varphi_{i}} D_{i}=\bigoplus_{i=0}^{s+t} \mathcal{A}_{\mu(i)} \square_{\varphi_{\mu(i)}} D_{\mu(i)} .
$$

Then by Theorem 2.4(iii), we conclude that $\mathcal{D}$ is a left $G_{(m, 3, r)}$-code over $\mathbb{F}_{q}$.

$\diamond$ First, we give the clear expression of $D_{\mu(i)}=\mu\left(B_{i}\right)$.

For the trivial case: $B_{i}=\mathcal{R}_{i}$ or $B_{i}=\{0\}$, the conclusion follows from Lemma 4.2(v) immediately. Then we only need to consider the nontrivial cases in $(\mathrm{A})$ and $(\mathrm{B})$.

In the case of (A-1), $B_{i}=\frac{y^{3}-1}{g(y)} \mathcal{R}_{i}$. If $g(y)=(y-1)^{2}$, then $B_{i}=(y-1) \mathcal{R}_{i}$. By Lemma 4.2 $(\mathrm{v})$ and Lemma 4.4 (i), we have $D_{\mu(i)}=\mu\left(B_{i}\right)=\mathcal{R}_{\mu(i)} \mu(y-1)=\mathcal{R}_{\mu(i)}(y-1)$.

Similarly, one can easily prove that the other conclusions in (i-1) and all conclusions in (i-2) hold from (A-1) and (A-2).

In the case of (A-3), $B_{i}=\frac{y^{3}-1}{g(y)} \mathcal{R}_{i}$. If $g(y)=(y-1)\left(y-\omega(x)^{2}\right)$, by Lemma 4.2(v) and Lemma 4.4 (ii), we have

$$
\begin{aligned}
D_{\mu(i)} & =\mu\left(\left(y-\omega_{i}(x)\right) \mathcal{R}_{i}\right)=\mu\left(\mathcal{R}_{i}\right) \mu\left(\left(y-\omega_{i}(x)\right)\right. \\
& =\mathcal{R}_{\mu(i)}\left(y-\omega_{i}\left(x^{-1}\right)^{2}\right) .
\end{aligned}
$$

Similarly, one can easily prove that the other conclusions in (i-3) hold from (A-3).

In the case of (B-1), by Lemma 4.2(v) and Lemma 4.4(iii) we have

$$
\begin{aligned}
D_{\mu(i)} & =\mu\left((-\alpha(x)+y) \mathcal{R}_{i}\right)=\mu\left(\mathcal{R}_{i}\right) \mu(-\alpha(x)+y) \\
& =\mathcal{R}_{\mu(i)}\left(-\theta_{\mu(i)}(\widehat{\alpha}(x))+y\right) \\
& =C_{\mu(i), 2, \theta_{\mu(i)}(\widehat{\alpha})}
\end{aligned}
$$

Hence the conclusion (ii-1) holds by Theorem 3.3 (ii).

Similarly, in the case of (B-2) we have

$$
\begin{aligned}
& D_{\mu(i)}=\mu\left(\mathcal{R}_{i}\right) \mu\left(\alpha(x)^{-1}+\theta_{i}^{2}(\alpha(x)) y+y^{2}\right) \\
= & \mathcal{R}_{\mu(i)}\left(\left(\theta_{\mu(i)}^{2}(\widehat{\alpha}(x))\right)^{-1}+\theta_{\mu(i)}^{2}\left(\theta_{\mu(i)}^{2}(\widehat{\alpha}(x))\right) y+y^{2}\right) \\
= & C_{\mu(i), 1, \theta_{\mu(i)}^{2}(\widehat{\alpha}) .}
\end{aligned}
$$

Hence the conclusion (ii-2) holds by Theorem 3.3 (iii).

$\diamond$ Then we prove that $|\mathcal{C}||\mathcal{D}|=\left|\mathbb{F}_{q}\right|^{3 m}$. For any $0 \leq i \leq s+t$, by Theorems 3.1, 3.3 and direct calculations we deduce that $\left|C_{i}\right|\left|D_{\mu(i)}\right|=\left|K_{i}\right|^{3}=\left|\mathcal{R}_{i}\right|$. From this and by Theorem 2.4 (i)-(iii), we obtain

$$
\begin{aligned}
|\mathcal{C} \| \mathcal{D}| & =\left(\prod_{i=0}^{s+t}\left|C_{i}\right|\right)\left(\prod_{i=0}^{s+t}\left|D_{\mu(i)}\right|\right)=\prod_{i=0}^{s+t}\left|C_{i}\right|\left|D_{\mu(i)}\right| \\
& =\prod_{i=0}^{s+t}\left|\mathcal{R}_{i}\right|=\prod_{i=0}^{s+t}\left|\mathcal{A}_{i}\left[y ;\left.\theta\right|_{\mathcal{A}_{i}}\right] /\left\langle\varepsilon_{i}(x)\left(y^{3}-1\right)\right\rangle\right| \\
& =\left|\mathcal{A}[y ; \theta] /\left\langle y^{3}-1\right\rangle\right|=\left|\mathbb{F}_{q}\left[G_{(m, 3, r)}\right]\right| \\
& =\left|\mathbb{F}_{q}\right|^{3 m} .
\end{aligned}
$$

$\diamond$ We claim that $\mathcal{D} \subseteq \mathcal{C}^{\perp_{E}}$. In fact, let $\xi \in \mathcal{D}$ and $\eta \in \mathcal{C}$. Then for each integer $i, 0 \leq i \leq s+t$, there exist $\alpha_{i} \in C_{i}$ and $\beta_{i} \in D_{i}$ such that $\xi=\sum_{i=0}^{s+t} \varepsilon_{i}(x) \alpha_{i}$ and $\eta=\sum_{i=0}^{s+t} \varepsilon_{i}(x) \beta_{i}$, where $C_{i}$ and $D_{i}$ are left ideals of $\mathcal{R}_{i}$ given by (i)-(ii) and $\varepsilon_{i}(x) \alpha_{i}, \varepsilon_{i}(x) \beta_{i} \in \mathcal{A}_{i}\left[y ;\left.\theta\right|_{\mathcal{A}_{i}}\right] /\left\langle\varepsilon_{i}(x)\left(y^{3}-1\right)\right\rangle$. By Lemma 2.3 (iv), we see that $\varepsilon_{i}(x)$ is the 
multiplicative identity of $\mathcal{A}_{i}\left[y ;\left.\theta\right|_{\mathcal{A}_{i}}\right] /\left\langle\varepsilon_{i}(x)\left(y^{3}-1\right)\right\rangle$. Since $\varepsilon_{i}(x) \varepsilon_{j}(x)=0$ for all $0 \leq i \neq j \leq s+t$, we have $\varepsilon_{i}(x) \varepsilon_{\mu(j)}(x)=0$ if $i \neq \mu(j)$, i.e., $j \neq \mu(i)$. Hence

$$
\begin{aligned}
\xi \cdot \mu(\eta)= & \left(\sum_{i=0}^{s+t} \varepsilon_{i}(x) \alpha_{i}\right)\left(\sum_{i=0}^{s+t} \mu\left(\varepsilon_{i}(x) \beta_{i}\right)\right. \\
= & \left(\sum_{i=0}^{s+t}\left(\varepsilon_{i}(x) \alpha_{i}\right) \varepsilon_{i}(x)\right) \\
& \cdot\left(\sum_{i=0}^{s+t} \varepsilon_{\mu(i)}(x)\left(\mu\left(\beta_{i}\right) \varepsilon_{\mu(i)}(x)\right)\right) \\
= & \sum_{i, j=0}^{s+t}\left(\varepsilon_{i}(x) \alpha_{i}\right) \varepsilon_{i}(x) \varepsilon_{\mu(j)}(x)\left(\mu\left(\beta_{j}\right) \varepsilon_{\mu(j)}(x)\right) \\
= & \sum_{i=0}^{s+t}\left(\varepsilon_{i}(x) \alpha_{i}\right) \varepsilon_{i}(x)\left(\mu\left(\beta_{\mu(i)}\right) \varepsilon_{i}(x)\right) \\
= & \sum_{i=0}^{s+t} \varepsilon_{i}(x)\left(\alpha_{i} \mu\left(\beta_{\mu(i)}\right)\right) .
\end{aligned}
$$

Since $\beta_{\mu(i)} \in D_{\mu(i)}$, by Lemma 4.2 (i) we see that

$$
\mu\left(\beta_{\mu(i)}\right) \in \mu\left(D_{\mu(i)}\right)=\mu\left(\mu\left(B_{i}\right)\right)=B_{i} .
$$

From this and by (8), we deduce that $\alpha_{i} \mu\left(\beta_{\mu(i)}\right)=0$ for all $i=0,1, \ldots, s+t$, which implies $\xi \cdot \mu(\eta)=0$, and so $[\xi, \eta]_{E}=0$ by Lemma 4.1 (ii). Therefore, $\mathcal{D} \subseteq \mathcal{C}^{\perp_{E}}$.

As stated above, we conclude that $\mathcal{D}=\mathcal{C}^{\perp_{E}}$ since both $\mathcal{C}$ and $\mathcal{D}$ are linear codes over $\mathbb{F}_{q}$ of length $3 \mathrm{~m}$.

Finally, we determine self-orthogonal left $G_{(m, 3, r)}$-codes.

Theorem 4.6: All distinct self-orthogonal left $G_{(m, 3, r)}$-codes over $\mathbb{F}_{q}$ are given by the following

$$
\mathcal{C}=\bigoplus_{i=0}^{s+t} \mathcal{A}_{i} \square_{\varphi_{i}} C_{i}=\bigoplus_{i=0}^{s+t}\left\{\varepsilon_{i}(x) \xi \mid \xi \in C_{i}\right\}\left(\bmod x^{m}-1\right),
$$

where $C_{i}$ is an left ideal of $\mathcal{R}_{i}=K_{i}\left[y ; \theta_{i}\right] /\left\langle y^{3}-1\right\rangle$ given by one of the following four cases:

(i) $0 \leq i \leq s_{1}$. In this case, $C_{i}$ is given by one of the following three subcases.

(i-1) If $q \equiv 0(\bmod 3), C_{i}=\{0\}$ or $C_{i}=\mathcal{R}_{i}(y-1)^{2}$.

(i-2) If $q^{d_{i}} \equiv 2(\bmod 3), C_{i}=\{0\}$.

(i-3) Let $q^{d_{i}} \equiv 1(\bmod 3)$. If $\omega_{i}\left(x^{-1}\right) \equiv \omega_{i}(x)\left(\bmod f_{i}(x)\right)$, then $C_{i}=\{0\}, C_{i}=\mathcal{R}_{i}(y-1)\left(y-\omega_{i}(x)\right)$ or $C_{i}=\mathcal{R}_{i}(y-1)\left(y-\omega_{i}(x)^{2}\right)$. Otherwise, $C_{i}=\{0\}$.

(ii) $s_{1}+1 \leq i \leq s_{1}+s_{2}$. In this case, $C_{i}=\mathcal{R}_{i} g(y), C_{i+s_{2}}=\mathcal{R}_{i+s_{2}} \vartheta(y)$ and the pair $(g(y), \vartheta(y))$ of polynomials is given by one of the following three subcases.

(ii-1) Let $q \equiv 0(\bmod 3)$. There are 10 pairs $(g(y), \vartheta(y))$ :

\begin{tabular}{l|l}
\hline$g(y)$ & $\vartheta(y)$ \\
\hline$y^{3}-1$ & $y^{3}-1,(y-1)^{2}, y-1,1$ \\
$(y-1)^{2}$ & $y^{3}-1,(y-1)^{2}, y-1$ \\
$y-1$ & $y^{3}-1,(y-1)^{2}$ \\
1 & $y^{3}-1$ \\
\hline
\end{tabular}

(ii-2) Let $q^{d_{i}} \equiv 2(\bmod 3)$. There are 9 pairs $(g(y), \vartheta(y))$ :

\begin{tabular}{l|l}
\hline$g(y)$ & $\vartheta(y)$ \\
\hline$y^{3}-1$ & $y^{3}-1, y^{2}+y+1, y-1,1$ \\
$y^{2}+y+1$ & $y^{3}-1, y-1$ \\
$y-1$ & $y^{3}-1, y^{2}+y+1$ \\
1 & $y^{3}-1$ \\
\hline
\end{tabular}


(ii-3) Let $q^{d_{i}} \equiv 1(\bmod 3)$. There are 27 pairs $(g(y), \vartheta(y))$ :

\begin{tabular}{l|l}
\hline$g(y)$ & $\vartheta(y)\left(\bmod f_{\mu(i)}(x)\right)$ \\
\hline$y^{3}-1$ & $1, y^{2}+y+1,(y-1)\left(y-\omega_{i}\left(x^{-1}\right)\right)$, \\
& $(y-1)\left(y-\omega_{i}\left(x^{-1}\right)^{2}\right), y-1$, \\
& $y-\omega_{i}\left(x^{-1}\right)^{2}, y-\omega_{i}\left(x^{-1}\right), y^{3}-1$ \\
\hline$y^{2}+y+1$ & $y^{3}-1,(y-1)\left(y-\omega_{i}\left(x^{-1}\right)\right)$, \\
& $(y-1)\left(y-\omega_{i}\left(x^{-1}\right)^{2}\right), y-1$ \\
\hline$(y-1)(y-\omega(x))$ & $y^{3}-1,(y-1)\left(y-\omega_{i}\left(x^{-1}\right)\right)$, \\
& $y^{2}+y+1, y-\omega_{i}\left(x^{-1}\right)$ \\
\hline$(y-1)\left(y-\omega(x)^{2}\right)$ & $y^{3}-1,(y-1)\left(y-\omega_{i}\left(x^{-1}\right)^{2}\right)$, \\
& $y^{2}+y+1, y-\omega_{i}\left(x^{-1}\right)^{2}$ \\
\hline$y-1$ & $y^{3}-1, y^{2}+y+1$ \\
$y-\omega(x)$ & $y^{3}-1,(y-1)\left(y-\omega_{i}\left(x^{-1}\right)\right)$ \\
$y-\omega(x)^{2}$ & $y^{3}-1,(y-1)\left(y-\omega_{i}\left(x^{-1}\right)^{2}\right)$ \\
1 & $y^{3}-1$ \\
\hline
\end{tabular}

(iii) $s+1 \leq i \leq s+t_{1}$. In this case, $C_{i}=\{0\}$ or $C_{i}=C_{i, 1, \alpha}=\mathcal{R}_{i}\left(\alpha(x)^{-1}+\theta_{i}^{2}(\alpha(x)) y+y^{2}\right)$ where $\alpha=\alpha(x) \in \mathcal{G}_{i}$ satisfying

$$
\theta_{i}(\widehat{\alpha}) \theta_{i}^{2}(\widehat{\alpha}) \alpha+\theta_{i}(\widehat{\alpha}) \alpha \theta_{i}^{2}(\alpha)+1=0 .
$$

(iv) $s+t_{1}+1 \leq i \leq s+t_{1}+t_{2}$. In this case, there are exactly $10+8 q^{\frac{d_{i}}{3}}+8 q^{\frac{2 d_{i}}{3}}+q^{d_{i}}$ pairs $\left(C_{i}, C_{i+t_{2}}\right)$ given by one of the following four subcases:

(iv-1) $4+2 q^{\frac{d_{i}}{3}}+2 q^{\frac{2 d_{i}}{3}}$ pairs: $\left(\{0\}, C_{i+t_{2}}\right)$, where $C_{i+t_{2}}$ is any left ideal of $\mathcal{R}_{i+t_{2}}$ listed by Theorem 3.3

(iv-2) $\left(1+q^{\frac{d_{i}}{3}}+q^{\frac{2 d_{i}}{3}}\right)\left(3+q^{\frac{d_{i}}{3}}\right)$ pairs: $\left(C_{i, 1, \alpha}, C_{i+t_{2}}\right)$, where $\alpha=\alpha(x) \in \mathcal{G}_{i}$ and $C_{i+t_{2}}$ is one of the following $3+q^{\frac{d_{i}}{3}}$ left ideals of $\mathcal{R}_{i+t_{2}}$ :

- $C_{i+t_{2}}=\{0\}$.

- $C_{i+t_{2}}=C_{i+t_{2}, 1, \beta}$ where $\beta=\theta_{i+t_{2}}^{2}\left(\gamma\left(x^{-1}\right)^{-1}\right)\left(\bmod f_{i+t_{2}}(x)\right)$ and $\gamma(x) \in \mathcal{G}_{i}$ satisfying the following conditions:

$\phi_{i, \alpha}(\gamma(x))=0$, if $\theta_{i}(a(x))=a(x)^{q^{\frac{d_{i}}{3}}}$ for all $a(x) \in K_{i}$;

$\psi_{i, \alpha}(\gamma(x))=0$, if $\theta_{i}(a(x))=a(x)^{q^{2 \frac{d_{i}}{3}}}$ for all $a(x) \in K_{i}$.

- $C_{i+t_{2}}=C_{i+t_{2}, 2, \beta}$ where $\beta=\theta_{i+t_{2}}\left(\alpha\left(x^{-1}\right)^{-1}\right)\left(\bmod f_{i+t_{2}}(x)\right)$.

(iv-3) $2\left(1+q^{\frac{d_{i}}{3}}+q^{\frac{2 d_{i}}{3}}\right)$ pairs: $\left(C_{i, 2, \alpha}, C_{i+t_{2}}\right)$, where $\alpha=\alpha(x) \in \mathcal{G}_{i}$ and $C_{i+t_{2}}$ is one of the following 2 left ideals of $\mathcal{R}_{i+t_{2}}$ :

- $C_{i+t_{2}}=\{0\}$.

- $C_{i+t_{2}}=C_{i+t_{2}, 1, \beta}$ where $\beta=\theta_{i+t_{2}}^{2}\left(\alpha\left(x^{-1}\right)^{-1}\right)\left(\bmod f_{i+t_{2}}(x)\right)$.

(iv-1) 1 pair: $\left(\mathcal{R}_{i},\{0\}\right)$.

Proof: By Theorem 4.5 and its proof we have $\mathcal{C}^{\perp_{E}}=\oplus_{i=0}^{s+t} \mathcal{A}_{i} \square_{\varphi_{i}} D_{i}$, where $D_{i}=\mu\left(B_{\mu(i)}\right)$. From this and by Theorem 2.4, we deduce that $\mathcal{C}$ is a self-orthogonal left $G_{(m, 3, r)}$-code over $\mathbb{F}_{q}$ if and only if $C_{i} \subseteq D_{i}=\mu\left(B_{\mu(i)}\right)$ for all $i=0,1, \ldots, s+t$.

By Equation (8) and the proof of Theorem 4.5, it follows that $C_{\mu(i)} \cdot B_{\mu(i)}=\{0\}$ and

$$
\left|C_{\mu(i)}\right|\left|B_{\mu(i)}\right|=\left|C_{\mu(i)}\right|\left|\mu\left(B_{\mu(i)}\right)\right|=\left|C_{\mu(i)}\right|\left|D_{i}\right|=\left|\mathcal{R}_{\mu(i)}\right|,
$$

which implies that $C_{\mu(i)}$ is the annihilating left ideal of $B_{\mu(i)}$ in $\mathcal{R}_{\mu(i)}$, i.e.,

$$
C_{\mu(i)}=\operatorname{Ann}_{\mathcal{R}_{\mu(i)}}^{(L)}\left(B_{\mu(i)}\right)
$$

where $\operatorname{Ann}_{\mathcal{R}_{\mu(i)}^{(L)}}^{\left(B_{\mu(i)}\right)}=\left\{\xi \in \mathcal{R}_{\mu(i)} \mid \xi \eta=0, \forall \eta \in B_{\mu(i)}\right\}$.

Since $\mu$ is an $\mathbb{F}_{q}$-algebra anti-isomorphism from $\mathcal{R}_{i}$ onto $\mathcal{R}_{\mu(i)}$, by $C_{i} \cdot B_{i}=\{0\}$ and $\left|C_{i}\right|\left|B_{i}\right|=\left|\mathcal{R}_{i}\right|$ we have $\mu\left(B_{i}\right) \cdot \mu\left(C_{i}\right)=\{0\}$ and $\left|\mu\left(B_{i}\right)\right|\left|\mu\left(C_{i}\right)\right|=\left|\mathcal{R}_{\mu(i)}\right|$, which implies

$$
\operatorname{Ann}_{\mathcal{R}_{\mu(i)}}^{(L)}\left(\mu\left(C_{i}\right)\right)=\mu\left(B_{i}\right)=D_{\mu(i)} .
$$


From this, by $D_{i}=\mu\left(B_{\mu(i)}\right)$ and (9), we deduce that

$$
\begin{aligned}
C_{i} \subseteq D_{i} & \Longleftrightarrow B_{\mu(i)} \supseteq \mu\left(C_{i}\right) \\
& \Longleftrightarrow \operatorname{Ann}_{\mathcal{R}_{\mu(i)}\left(B_{\mu(i)}\right) \subseteq \operatorname{Ann}_{\mathcal{R}_{\mu(i)}}^{(L)}\left(\mu\left(C_{i}\right)\right)} \\
& \Longleftrightarrow C_{\mu(i)} \subseteq D_{\mu(i)}
\end{aligned}
$$

for all $i=0,1, \ldots, s+t$.

$\diamond$ Let $0 \leq i \leq s$. Then $\theta_{i}=\operatorname{id}_{K_{i}}$ and that $\mathcal{R}_{i}=K_{i}[y] /\left\langle y^{3}-1\right\rangle$ is a commutative ring. By Theorem 4.5 and its proof there is a unique pair $(g(y), h(y))$ of monic factors $g(y), h(y)$ of $y^{3}-1$ in $K_{i}[y]$ such that

$C_{i}=\mathcal{R}_{i} g(y), D_{i}=\mathcal{R}_{i} \frac{y^{3}-1}{h(y)}=\mu\left(B_{\mu(i)}\right)$ with $B_{\mu(i)}=\mathcal{R}_{\mu(i)} \mu\left(\frac{y^{3}-1}{h(y)}\right)$, and

$$
C_{\mu(i)}=\operatorname{Ann}_{\mathcal{R}_{\mu(i)}}^{(L)}\left(B_{\mu(i)}\right)=\mathcal{R}_{\mu(i)} \mu(h(y)) .
$$

Hence

$$
C_{i} \subseteq D_{i} \Longleftrightarrow \frac{y^{3}-1}{h(y)}\left|g(y) \Longleftrightarrow\left(y^{3}-1\right)\right| g(y) h(y) .
$$

By Lemma 4.2 (ii) and Theorems 3.1, we have one of the following two cases.

(i) When $0 \leq i \leq s_{1}, \mu(i)=i$, which implies $\mu(h(y)) \sim_{l} g(y)$ by (10), and so $\mu(g(y)) \sim_{l} h(y)$. Form this and by (11) we deduce that $C_{i} \subseteq D_{i}$ if and only if $\left(y^{3}-1\right) \mid g(y) \mu(g(x))$. Then the conclusions follow from Lemma 4.4 (i) and (ii).

(ii) Let $s_{1}+1 \leq i \leq s_{1}+s_{2}, \mu(i)=i+s_{2}$. By Lemma 4.4 (i) and (ii), we see that for each monic factor $h(y)$ of $y^{3}-1$ in $K_{i}[y]$ there is a unique monic factor $\vartheta(y)$ of $y^{3}-1$ in $K_{\mu(i)}[y]$ such that $\mu(h(y)) \sim_{l} \vartheta(y)$, which implies $C_{i+s_{2}}=\mathcal{R}_{\mu(i)} \vartheta(y)$ by (10). Then the conclusions follow from (11), Lemma 4.4 (i) and (ii) immediately.

$\diamond$ Let $s+1 \leq i \leq s+t$. Then $\mathcal{R}_{i}=K_{i}\left[y ; \theta_{i}\right] /\left\langle y^{3}-1\right\rangle$ is a noncommutative ring. By Theorem 4.5 (ii), the pair $\left(C_{i}, D_{\mu(i)}\right)$ is given by one of the following cases:

$\diamond C_{i}=\{0\}$ and $D_{\mu(i)}=\mathcal{R}_{\mu(i)}$, or $C_{i}=\mathcal{R}_{i}$ and $D_{\mu(i)}=\{0\}$;

$\diamond C_{i}=C_{i, 1, \alpha}$ and $D_{\mu(i)}=C_{\mu(i), 2, \theta_{\mu(i)}(\widehat{\alpha})}$, where $\alpha \in \mathcal{G}_{i}$;

$\diamond C_{i}=C_{i, 2, \alpha}$ and $D_{\mu(i)}=C_{\mu(i), 1, \theta_{\mu(i)}^{2}(\widehat{\alpha})}$, where $\alpha \in \mathcal{G}_{i}$.

From these, we deduce that

$$
\operatorname{dim}_{K_{i}}\left(C_{i}\right)+\operatorname{dim}_{K_{\mu(i)}}\left(C_{\mu(i)}\right)=3 .
$$

Then by Lemma 4.2(ii), we have one and only one of the following two cases.

(iii) Let $s+1 \leq i \leq s+t_{1}$. Then $\mu(i)=i$. In this case, we deduce that the condition $C_{i} \subseteq D_{i}$ if and only if $C_{i}=\{0\}$ or $C_{i}=C_{i, 1, \alpha}$ where $\alpha=\alpha(x) \in \mathcal{G}_{i}$ satisfying $C_{i, 1, \alpha} \subset C_{i, 2, \theta_{i}(\widehat{\alpha})}$. By Theorem 3.6 (i), the condition $C_{i, 1, \alpha} \subset C_{i, 2, \theta_{i}(\widehat{\alpha})}$ is equivalent to that $\theta_{i}(\widehat{\alpha}) \theta_{i}\left(\theta_{i}(\widehat{\alpha})\right) \alpha+\theta_{i}(\widehat{\alpha}) \alpha \theta_{i}^{2}(\alpha)+1=0$, i.e., $\theta_{i}(\widehat{\alpha}) \theta_{i}^{2}(\widehat{\alpha}) \alpha+\theta_{i}(\widehat{\alpha}) \alpha \theta_{i}^{2}(\alpha)+1=$ 0 .

(iv) Let $s+t_{1}+1 \leq i \leq s+t_{1}+t_{2}$. Then $\mu(i)=i+t_{2}$. By Theorem 4.5(ii), we have one of the following four situations:

(iv-1) Let $C_{i}=\{0\}$. Then $D_{i+t_{2}}=\mathcal{R}_{\mu(i)}$. In this case, $C_{i+t_{2}} \subseteq D_{i+t_{2}}$ for any left ideal $C_{i+t_{2}}$ of $\mathcal{R}_{\mu(i)}$.

By Theorem 3.3, the number of pairs $\left(\{0\}, C_{i+t_{2}}\right)$ is equal to $4+2 q^{\frac{d_{i}}{3}}+2 q^{\frac{2 d_{i}}{3}}$.

(iv-2) Let $C_{i}=C_{i, 1, \alpha}$ where $\alpha=\alpha(x) \in \mathcal{G}_{i}$. Then $\operatorname{dim}_{K_{i+t_{i}}}\left(D_{i+t_{2}}\right)=2$ by (12) and

$$
D_{i+t_{2}}=C_{i+t_{2}, 2, \theta_{i+t_{2}}(\widehat{\alpha})}
$$

by Theorem 4.5 (ii-1). Hence $\operatorname{dim}_{K_{i+t_{i}}}\left(C_{i+t_{2}}\right) \leq 2$ if $C_{i+t_{2}} \subseteq D_{i+t_{2}}$. Then we have one of the following three cases.

$\triangleright$ It is obvious that $C_{i+t_{2}}=\{0\}$ satisfying $C_{i+t_{2}} \subseteq D_{i+t_{2}}$.

$\triangleright$ Let $C_{i+t_{2}}=C_{i+t_{2}, 1, \beta}$ where $\beta=\beta(x) \in \mathcal{G}_{i+t_{2}}$. By Lemma 4.2 (ii), we have $\mu\left(i+t_{2}\right)=i$, which implies $D_{i}=D_{\mu\left(i+t_{2}\right)}=C_{i, 2, \theta_{i}(\widehat{\beta})}$ by Theorem 4.5(ii-1). From this and by Theorem 3.6(ii), we deduce that $C_{i} \subset D_{i}$ if and only if $\theta_{i}(\widehat{\beta})$ satisfies the following conditions:

$$
\begin{aligned}
& \phi_{i, \alpha}\left(\theta_{i}(\widehat{\beta})\right)=0, \text { if } \theta_{i}(a(x))=a(x)^{q^{\frac{d_{i}}{3}}} \text { for all } a(x) \in K_{i} . \\
& \psi_{i, \alpha}\left(\theta_{i}(\widehat{\beta})\right)=0 \text {, if } \theta_{i}(a(x))=a(x)^{q^{\frac{2 d_{i}}{3}}} \text { for all } a(x) \in K_{i} .
\end{aligned}
$$


We denote $\gamma=\gamma(x)=\theta_{i}(\widehat{\beta}(x))$. Then $\gamma=\theta_{i}\left(\mu(\beta(x))^{-1}\right) \in \mathcal{G}_{i}$ by Corollary 4.3, which implies $\mu(\beta(x))^{-1}=$ $\theta_{i}^{2}(\gamma(x))$, and hence $\beta(x)=\theta_{i+t_{2}}^{2}\left(\gamma\left(x^{-1}\right)^{-1}\right)$ by Lemma 4.2 (iv). Moreover, by Lemma 3.5 we know that both $\phi_{i, \alpha}(x)$ and $\psi_{i, \alpha}(x)$ have exactly $q^{\frac{d_{i}}{3}}+1$ roots in $\mathcal{G}_{i}$.

$\triangleright$ Let $C_{i+t_{2}}=C_{i+t_{2}, 2, \beta}$ where $\beta=\beta(x) \in \mathcal{G}_{i+t_{2}}$. As $\mu\left(i+t_{2}\right)=i$, we have $D_{i}=D_{\mu\left(i+t_{2}\right)}=C_{i, 1, \theta_{i}^{2}(\widehat{\beta})}$ by Theorem 4.5 (ii-2). Hence $C_{i}=C_{i, 1, \alpha} \subseteq D_{i}$ if and only if $\alpha=\theta_{i}^{2}(\widehat{\beta})=\theta_{i}^{2}\left(\beta\left(x^{-1}\right)^{-1}\right)$, which is equivalent to that $\beta(x)=\theta_{i+t_{2}}\left(\alpha\left(x^{-1}\right)^{-1}\right)$ by Lemma 4.2(iv).

Therefore, the number of pairs $\left(C_{i, 1, \alpha}, C_{i+t_{2}}\right)$ is equal to

$$
\left(1+q^{\frac{d_{i}}{3}}+q^{\frac{2 d_{i}}{3}}\right)\left(3+q^{\frac{d_{i}}{3}}\right) .
$$

(iv-3) Let $C_{i}=C_{i, 2, \alpha}$ where $\alpha=\alpha(x) \in \mathcal{G}_{i}$. Then $\operatorname{dim}_{K_{i+t_{i}}}\left(D_{i+t_{2}}\right)=1$ by (12) and

$$
D_{i+t_{2}}=C_{i+t_{2}, 1, \theta_{i+t_{2}}^{2}(\widehat{\alpha})}
$$

by Theorem 4.5(ii-2). Hence $\operatorname{dim}_{K_{i+t_{i}}}\left(C_{i+t_{2}}\right) \leq 1$ if $C_{i+t_{2}} \subseteq D_{i+t_{2}}$. Then we have one of the following two cases. $\triangleright C_{i+t_{2}}=\{0\}$.

$\triangleright$ Let $C_{i+t_{2}}=C_{i+t_{2}, 1, \beta}$ where $\beta=\beta(x) \in \mathcal{G}_{i+t_{2}}$. Then As $\mu\left(i+t_{2}\right)=i$, we have $D_{i}=D_{\mu\left(i+t_{2}\right)}=C_{i, 2, \theta_{i}(\widehat{\beta})}$ by Theorem 4.5 (ii-1). Hence $C_{i}=C_{i, 2, \alpha} \subseteq D_{i}$ if and only if $\alpha=\theta_{i}(\widehat{\beta})=\theta_{i}^{2}\left(\beta\left(x^{-1}\right)^{-1}\right)$, which is equivalent to that $\beta(x)=\theta_{i+t_{2}}^{2}\left(\alpha\left(x^{-1}\right)^{-1}\right)$ by Lemma 4.2 (iv).

Therefore, the number of pairs $\left(C_{i, 1, \alpha}, C_{i+t_{2}}\right)$ is equal to $2\left(1+q^{\frac{d_{i}}{3}}+q^{\frac{2 d_{i}}{3}}\right)$.

(iv-4) Let $C_{i}=\mathcal{R}_{i}$. Then $D_{i+t_{2}}=\{0\}$. From this and by $C_{i+t_{2}} \subseteq D_{i+t_{2}}$, we deduce $C_{i+t_{2}}=\{0\}$.

As stated above, we conclude that the number of pairs $\left(C_{i}, C_{i+t_{2}}\right)$, where $s+t_{1}+1 \leq i \leq s+t_{1}+t_{2}$, is equal to $10+8 q^{\frac{d_{i}}{3}}+8 q^{\frac{2 d_{i}}{3}}+q^{d_{i}}$.

\section{AN EXAMPLE}

We consider left $G_{(14,3,9)}$-codes over $\mathbb{F}_{3}$. Obviously, $9^{3}=729 \equiv 1(\bmod 14)$. All distinct 3-cyclotomic cosets modulo 14 are the following: $J_{0}^{(3)}=\{0\}, J_{7}^{(3)}=\{7\}, J_{2}^{(3)}=\{2,6,4,12,8,10\}, J_{1}^{(3)}=\{1,3,9,13,11,5\}$. It is clear that

$$
\theta(7)=9 \cdot 7 \equiv 7, \theta(2)=9 \cdot 2 \equiv 4, \theta(1)=9(\bmod 14) .
$$

Using the notations is Section 2, we have that $s=1, t=2, J(0)=J_{0}^{(3)}, J(1)=J_{7}^{(3)}, J(2)=J_{2}^{(3)}$ and $J(3)=J_{1}^{(3)}$. Hence $d_{0}=d_{1}=1, d_{i}=6$ and $\frac{d_{i}}{3}=2$ for $i=2,3$.

Obviously, $3 \equiv 0(\bmod 3)$. By Corollary $3.4(\mathrm{i})$, the number of left $G_{(14,3,9)}$-codes over $\mathbb{F}_{3}$ is equal to

$$
4^{2} \prod_{i=2,3}\left(4+2 \cdot 3^{\frac{d_{i}}{3}}+2 \cdot 3^{\frac{2 d_{i}}{3}}\right)=16 \cdot 184^{2}=541,696
$$

We have $x^{14}-1=f_{0}(x) f_{1}(x) f_{2}(x) f_{3}(x)$, where $f_{0}(x)=x-1, f_{1}(x)=x+1, f_{2}(x)=x^{6}+x^{5}+x^{4}+x^{3}+$ $x^{2}+x+1$ and $f_{3}(x)=x^{6}+2 x^{5}+x^{4}+2 x^{3}+x^{2}+2 x+1$. Then

- $K_{0}=\mathbb{F}_{3}[x] /\langle x-1\rangle=\mathbb{F}_{3}$ and $\mathcal{R}_{0}=K_{0}[y] /\left\langle y^{3}-1\right\rangle=\mathbb{F}_{3}[y] /\left\langle(y-1)^{3}\right\rangle$. By Theorem 3.1 (ii), all distinct ideals of $\mathcal{R}_{0}$ are given by: $C_{0}=\mathcal{R}_{0} g(y)$, where $g(y) \in\left\{1, y-1,(y-1)^{2}, y^{3}-1\right\}$.

- $K_{1}=\mathbb{F}_{3}[x] /\langle x+1\rangle=\mathbb{F}_{3}$ and $\mathcal{R}_{1}=K_{1}[y] /\left\langle y^{3}-1\right\rangle=\mathbb{F}_{3}[y] /\left\langle(y-1)^{3}\right\rangle$. By Theorem 3.1 (ii), all distinct ideals of $\mathcal{R}_{1}$ are given by: $C_{1}=\mathcal{R}_{1} g(y)$, where $g(y) \in\left\{1, y-1,(y-1)^{2}, y^{3}-1\right\}$.

Moreover, $|\langle 0\rangle|=1,\left|\mathcal{R}_{0}\right|=\left|\mathcal{R}_{1}\right|=3^{3}=27,\left|\mathcal{R}_{0}(y-1)\right|=\left|\mathcal{R}_{1}(y-1)\right|=3^{2}=9$ and $\left|\mathcal{R}_{0}(y-1)^{2}\right|=$ $\left|\mathcal{R}_{1}(y-1)^{2}\right|=3$.

- $K_{2}=\mathbb{F}_{2}[x] /\left\langle f_{2}(x)\right\rangle=\left\{\sum_{j=0}^{5} a_{j} x^{j} \mid a_{j} \in \mathbb{F}_{3}, j=0,1, \ldots, 5\right\}$ and $\varrho_{2}(x)=1+x$ is an element of multiplicative order $1+3^{2}+3^{4}=91$ in $K_{2}$. Hence

$$
\mathcal{G}_{2}=\left\{(1+x)^{\lambda} \mid \lambda=0,1, \ldots, 90\right\}\left(\bmod f_{2}(x)\right)
$$

and $\mathcal{R}_{2}=K_{2}\left[y ; \theta_{2}\right] /\left\langle y^{3}-1\right\rangle$ where $\theta_{2}$ is an $\mathbb{F}_{3}$-algebra automorphism of $K_{2}$ defined by:

$$
\theta_{2}(a(x))=a\left(x^{r}\right)=a\left(x^{9}\right)=a(x)^{9}\left(\bmod f_{2}(x)\right)
$$

for all $a(x) \in K_{2}$. Then $\theta_{2}^{2}\left(\varrho_{2}(x)\right)=(1+x)^{9^{2}}=1+x^{4}$. 
By Theorem 3.3, all distinct left ideals $C_{2}$ of $\mathcal{R}_{2}$ are given by the following three cases:

(i) $C_{2}=\{0\}$ with $|\{0\}|=1$, and $C_{2}=\mathcal{R}_{2}$ with $\left|\mathcal{R}_{2}\right|=\left|K_{2}\right|^{3}=\left(3^{6}\right)^{3}=3^{18}=387420489$.

(ii) $C_{2}=C_{2,2,(1+x)^{\lambda}}=\mathcal{R}_{2}\left(-(1+x)^{\lambda}+y\right)$ with $\left|C_{2}\right|=\left(3^{6}\right)^{2}=3^{12}=531441, \lambda=0,1,2, \ldots, 90$.

(iii) $C_{2}=C_{2,1,(1+x)^{\lambda}}=\mathcal{R}_{2}\left((1+x)^{91-\lambda}+\left(1+x^{4}\right)^{\lambda} y+y^{2}\right)$ with $\left|C_{2}\right|=3^{6}=729, \lambda=0,1,2, \ldots, 90$.

- $K_{3}=\mathbb{F}_{2}[x] /\left\langle f_{3}(x)\right\rangle=\left\{\sum_{j=0}^{5} a_{j} x^{j} \mid a_{j} \in \mathbb{F}_{3}\right\}$. We find that $\varrho_{3}(x)=1+2 x$ is an element of multiplicative order $1+3^{2}+3^{4}=91$ in $K_{3}$. Hence

$$
\mathcal{G}_{3}=\left\{(1+2 x)^{\lambda} \mid \lambda=0,1, \ldots, 90\right\}\left(\bmod f_{3}(x)\right)
$$

and $\mathcal{R}_{3}=K_{3}\left[y ; \theta_{2}\right] /\left\langle y^{3}-1\right\rangle$ where $\theta_{3}$ is an $\mathbb{F}_{3}$-algebra automorphism of $K_{3}$ defined by:

$$
\theta_{3}(a(x))=a\left(x^{r}\right)=a\left(x^{9}\right)=a(x)^{9}\left(\bmod f_{3}(x)\right)
$$

for all $a(x) \in K_{3}$. In particular, we have $\theta_{3}^{2}\left(\varrho_{2}(x)\right)=(1+x)^{9^{2}}=1+x^{4}$.

By Theorem 3.3 , all distinct left ideals $C_{3}$ of $\mathcal{R}_{3}$ are given by the following three cases:

(i) $\{0\}$ and $\mathcal{R}_{3}$, where $|\{0\}|=1$ and $\left|\mathcal{R}_{3}\right|=\left|K_{3}\right|^{3}=\left(3^{6}\right)^{3}=3^{18}=387420489$.

(ii) $C_{3}=C_{3,2,(1+2 x)^{\lambda}}=\mathcal{R}_{3}\left(-(1+2 x)^{\lambda}+y\right)$ with $\left|C_{3}\right|=\left(3^{6}\right)^{2}=3^{12}=531441, \lambda=0,1,2, \ldots, 90$.

(iii) $C_{3}=C_{3,1,(1+x)^{\lambda}}=\mathcal{R}_{3}\left((1+2 x)^{91-\lambda}+\left(1+x^{4}\right)^{\lambda} y+y^{2}\right)$ with $\left|C_{3}\right|=3^{6}=729, \lambda=0,1,2, \ldots, 90$.

- All distinct 541696 left $G$-codes over $\mathbb{F}_{3}$ are given by

$$
\mathcal{C}=\bigoplus_{i=0}^{3} \mathcal{A}_{i} \square_{\varphi_{i}} C_{i}=\sum_{i=0}^{3}\left\{\varepsilon_{i}(x) \xi_{i} \mid \xi_{i} \in C_{i}\right\}
$$

$\left(\bmod x^{14}-1\right)$ by Theorem 2.4, where

$$
\begin{aligned}
& \varepsilon_{0}(x)=2+2 x+2 x^{2}+2 x^{3}+2 x^{4}+2 x^{5}+2 x^{6}+2 x^{7}+2 x^{8}+2 x^{9}+2 x^{10}+2 x^{11}+2 x^{12}+2 x^{13}, \\
& \varepsilon_{1}(x)=2+x+2 x^{2}+x^{3}+2 x^{4}+x^{5}+2 x^{6}+x^{7}+2 x^{8}+x^{9}+2 x^{10}+x^{11}+2 x^{12}+x^{13}, \\
& \varepsilon_{2}(x)=x+x^{2}+x^{3}+x^{4}+x^{5}+x^{6}+x^{8}+x^{9}+x^{10}+x^{11}+x^{12}+x^{13}, \\
& \varepsilon_{3}(x)=2 x+x^{2}+2 x^{3}+x^{4}+2 x^{5}+x^{6}+x^{8}+2 x^{9}+x^{10}+2 x^{11}+x^{12}+2 x^{13},
\end{aligned}
$$

and the number of codewords in $\mathcal{C}$ is equal to

$$
|\mathcal{C}|=\left|C_{0}\right|\left|C_{1}\right|\left|C_{2}\right|\left|C_{3}\right| \text {. }
$$

As $-J(i)=J(i)(\bmod 14)$, we have $\mu(i)=i$ for all $i=0,1,2,3$. Using the notations of Lemma 4.2, we have $s=s_{1}=1, s_{2}=0, t=t_{1}=2$ and $t_{2}=0$. Hence $\mu\left(\varepsilon_{i}(x)\right)=\varepsilon_{i}(x)$ for all $i=0,1,2,3$.

- By Theorem 4.6, all self-orthogonal left $G_{(14,3,9)}$-codes over $\mathbb{F}_{3}$ are given by: $\mathcal{C}=\bigoplus_{i=0}^{3} \mathcal{A}_{i} \square_{\varphi_{i}} C_{i}$, where

$\diamond C_{0}=\{0\}$ or $C_{0}=\mathcal{R}_{0}(y-1)^{2}$.

$\diamond C_{1}=\{0\}$ or $C_{1}=\mathcal{R}_{1}(y-1)^{2}$.

$\diamond C_{2}=\{0\}$ or $C_{2}=C_{2,1, \alpha(x)}$ where $\alpha(x)=(1+x)^{\lambda}$ satisfying $\theta_{i}(\widehat{\alpha}) \theta_{i}^{2}(\widehat{\alpha}) \alpha+\theta_{i}(\widehat{\alpha}) \alpha \theta_{i}^{2}(\alpha)+1=0$, i.e.,

$$
\begin{aligned}
0= & 1+\left(1+x^{-1}\right)^{-9 \lambda-81 \lambda}(1+x)^{\lambda} \\
& +\left(1+x^{-1}\right)^{-9 \lambda}(1+x)^{\lambda+81 \lambda} \\
= & 1+x^{90 \lambda}(1+x)^{-89 \lambda}+x^{9 \lambda}(1+x)^{73 \lambda}
\end{aligned}
$$

in $K_{2}(0 \leq \lambda \leq 90)$. Since $x^{14}=1$ and $(1+x)^{91}=1$ in $K_{2}$, the above condition is equivalent to

$$
1+x^{6 \lambda}(1+x)^{2 \lambda}+x^{9 \lambda}(1+x)^{73 \lambda} \equiv 0\left(\bmod f_{2}(x)\right) \text {. }
$$

Precisely, we have

$$
\lambda=0,7,8,11,13,20,21,24,26,33,34,37,39,46,47,
$$

$$
50,52,59,60,63,65,72,73,76,78,85,86,89 .
$$

$\diamond C_{3}=\{0\}$ or $C_{3}=C_{3,1,(1+2 x)^{\lambda}}$ where $\lambda$ is given by (13).

Therefore, the number of self-orthogonal left $G_{(14,3,9)}$-codes over $\mathbb{F}_{3}$ is equal to $2 \cdot 2 \cdot 29 \cdot 29=3364$.

For example, we have 21 self-orthogonal left $G_{(14,3,9)}$-codes over $\mathbb{F}_{3}: \mathcal{C}=\left\{\varepsilon_{2}(x) \xi \mid \xi \in C_{2,1,(1+x)^{\lambda}}\right\}$ where $\lambda=$ $7,8,11,20,21,24,33,34,37,46,47,50,59,60,63,72,73,76,85,86,89$, which are self-orthogonal linear $[42,6,18]$ codes over $\mathbb{F}_{3}$ with the following Hamming weight enumerator:

$$
W_{\mathcal{C}}(Y)=1+14 Y^{18}+294 Y^{24}+336 Y^{30}+84 Y^{36} \text {. }
$$




\section{CONCLUSION}

Let $G_{(m, 3, r)}$ be a metacyclic group of order $3 m, r \equiv q^{\epsilon}(\bmod m)$ for some positive integer $\epsilon$ and $\operatorname{gcd}(m, q)=1$. We present a system theory of left $G_{(m, 3, r)}$-codes over $\mathbb{F}_{q}$, only using finite field theory and basic theory of cyclic codes and skew cyclic codes. We prove that any left $G_{(m, 3, r)}$-code is a direct sum of concatenated codes with inner codes $\mathcal{A}_{i}$ and outer codes $C_{i}$, where $\mathcal{A}_{i}$ is a minimal cyclic code over $\mathbb{F}_{q}$ of length $m$ and $C_{i}$ is a skew cyclic code of length 3 over an extension field of $\mathbb{F}_{q}$, and provide an explicit expression for each outer code in every concatenated code. Moreover, we give the dual code of each left $G_{(m, 3, r)}$-code and determine all self-orthogonal left $G_{(m, 3, r)}$-codes over $\mathbb{F}_{q}$.

\section{ACKNOWLEDGMENT}

Part of this work was done when Yonglin Cao was visiting Chern Institute of Mathematics, Nankai University, Tianjin, China. Yonglin Cao would like to thank the institution for the kind hospitality. This research is supported in part by the National Natural Science Foundation of China (Grant Nos. 11471255, 61171082, 61571243) and the National Key Basic Research Program of China (Grant No. 2013CB834204).

\section{REFERENCES}

[1] Boucher D., Geiselmann W. Ulmer F.: Skew cyclic codes. Appl. Algebra in Engrg. Comm. Comput. 18, 379-389 (2007).

[2] Boucher D., Solé P. Ulmer F.: Skew constacyclic codes over Galois rings. Adv. Math. Commun. 2, $273-292$ (2008).

[3] Boucher D., Ulmer F.: Codes as modules over skew polynomial rings. Lecture Notes in Comput. Sci. 5921, 38-55 (2009).

[4] Boucher D., Ulmer F.: Coding with skew polynomial rings. J. Symbolic Comput. 44. 1644-1656 (2009).

[5] Gabriela O., Inneke V. G.: Construction of minimal non-abelian left group codes. Des. Codes Cryptogr. 75, 359-373 (2015).

[6] Huffman W. C., Pless V.: Fundamentals of error correcting codes. Cambridge University Press 2003.

[7] Jitman S., Ling S., Udomkavanich P.: Skew constacyclic codes over finite chain rings. Adv. Math. Commun. 6, 39-63 (2012).

[8] Jitman S., Ling S., Liu H., Xie X.: Abelian codes in principal ideal group algebras, IEEE Trans. Inf. Theory 59 (5), $3046-3058$ (2013).

[9] Passman D. S.: The Algebraic Structure of Group Rings. Wiley, New York (1977).

[10] Sabin R., Lomonaco S.: Metacyclic error-correcting codes. Appl. Algebra in Engrg. Comm. Comput. 6, 191-210 (1995).

[11] Sendrier N.: On the concatented structure of a linear code. Appl. Algebra in Engrg. Comm. Comput. 9, $221-242$ (1998). 